\title{
Diffusive Mixing of Stable States in the Ginzburg-Landau Equation
}

\author{
Thierry Gallay* and Alexander Mielke ${ }^{\dagger}$
}

January 22, 1998

\begin{abstract}
The Ginzburg-Landau equation $\partial_{t} u=\partial_{x}^{2} u+u-|u|^{2} u$ on the real line has spatially periodic steady states of the the form $U_{\eta, \beta}(x)=\sqrt{1-\eta^{2}} \mathrm{e}^{\mathrm{i}(\eta x+\beta)}$, with $|\eta| \leq 1$ and $\beta \in \mathbb{R}$. For $\eta_{+}, \eta_{-} \in\left(-\frac{1}{\sqrt{3}}, \frac{1}{\sqrt{3}}\right), \beta_{+}, \beta_{-} \in \mathbb{R}$, we construct solutions which converge for all $t>0$ to the limiting pattern $U_{\eta_{ \pm}, \beta_{ \pm}}$as $x \rightarrow \pm \infty$. These solutions are stable with respect to sufficiently small $\mathrm{H}^{2}$ perturbations, and behave asymptotically in time like $\left(1-\widetilde{\eta}(x / \sqrt{t})^{2}\right)^{1 / 2} \exp (\mathrm{i} \sqrt{t} \widetilde{N}(x / \sqrt{t}))$, where $\widetilde{N}^{\prime}=\widetilde{\eta} \in \mathcal{C}^{\infty}(\mathbb{R})$ is uniquely determined by the boundary conditions $\widetilde{\eta}( \pm \infty)=\eta_{ \pm}$. This extends a previous result of [BrK92] by removing the assumption that $\eta_{ \pm}$should be close to zero. The existence of the limiting profile $\widetilde{\eta}$ is obtained as an application of the theory of monotone operators, and the long-time behavior of our solutions is controlled by rewriting the system in scaling variables and using energy estimates involving an exponentially growing damping term.
\end{abstract}

*Analyse Numérique et EDP, Université de Paris XI, Thierry.Gallay@math.u-psud.fr

$\dagger$ †nstitut für Angewandte Mathematik, Universität Hannover, mielke@ifam.uni-hannover.de 


\section{Introduction}

The dynamics of parabolic partial differential equations on unbounded spatial domains exhibit various interesting phenomena which are not present on bounded domains, since the semigroups associated to such extended systems are not compact. An important example is the development of so-called "side-band instabilities" for spatially periodic patterns bifurcating from a homogeneous steady state. Although this problem has been investigated at a formal level more than 30 years ago [Eck65], only recently a rigorous approach has been proposed and applied to various model problems, see [CoE90a], Mi97b and the references therein. In particular, nonlinear stability results have been obtained for the spatially periodic solutions of the one-dimensional Ginzburg-Landau [CEE92] and SwiftHohenberg Sch96 equations, as well as for the Taylor vortices in a cylindrical geometry Sch97. These periodic patterns appear to be stable with respect to sufficiently localized perturbations, and the convergence of the perturbed solutions towards the equilibrium state is only diffusive (typically $\mathcal{O}\left(t^{-1 / 2}\right)$ ) as $t \rightarrow+\infty$. In higher dimensions, we quote the recent results by [Mi97d] and [Uec98] which prove the linear and nonlinear stability of the roll solutions for the two-dimensional Swift-Hohenberg equation.

Following up the work of Collet-Eckmann [CoE92 and Bricmont-Kupiainen BrK92, we study here one particular aspect of the diffusive stability theory, namely the mixing of two spatially periodic steady states. Consider a partial differential equation on the real line having a continuous family of spatially periodic time-independent solutions, some of them being (diffusively) stable. For this system, we prescribe initial data which coincide with one stable pattern as $x \rightarrow-\infty$ and with another one as $x \rightarrow+\infty$. The question is to determine the long-time behavior of the solutions arising from such initial data.

As in CoE92, BrK92, we investigate this problem for the Ginzburg-Landau equation with real coefficients

$$
\partial_{t} u=\partial_{x}^{2} u+u-|u|^{2} u, \quad t \geq 0, \quad x \in \mathbb{R},
$$

where $u(t, x) \in \mathbb{C}$. This system has a family of periodic stationary states given by

$$
U_{\eta, \beta}(x)=\sqrt{1-\eta^{2}} \mathrm{e}^{\mathrm{i}(\eta x+\beta)},
$$

where $|\eta| \leq 1$ and $\beta \in \mathbb{R}$. These solutions are linearly stable if and only if $\eta^{2} \leq 1 / 3$ Eck65, and nonlinearly stable with respect to $\mathrm{H}^{1}$ perturbations for all $\eta^{2}<1 / 3$ [CEE92, Ga95. Thus, we shall study solutions of (1.1) satisfying

$$
u(t, x)-U_{\eta_{ \pm}, \beta_{ \pm}}(x) \rightarrow 0 \text { as } x \rightarrow \pm \infty
$$

with $\eta_{+}, \eta_{-} \in\left(-\frac{1}{\sqrt{3}}, \frac{1}{\sqrt{3}}\right)$ and $\beta_{+}, \beta_{-} \in \mathbb{R}$.

If $\eta_{+}=\eta_{-}$and $\beta_{+}=\beta_{-}$, the problem reduces to the diffusive stability of the steady state $U_{\eta_{-}, \beta_{-}}$. In the sequel, we shall concentrate on the most interesting case $\eta_{+} \neq \eta_{-}$, and make only a few comments on the intermediate situation where $\eta_{+}=\eta_{-}$and $\beta_{+} \neq \beta_{-}$. Thus, we assume that $\eta_{+} \neq \eta_{-}$and, without loss of generality, that $\beta_{+}=\beta_{-}=0$, see (2.1) below. If both $\eta_{+}, \eta_{-}$are close to zero, it is shown in BrK92 that, under technical assumptions on the initial data, the solutions of (1.1) satisfying (1.2) converge locally as $t \rightarrow+\infty$ to the circle of steady states $\mathbf{S}_{*}=\left\{U_{\eta_{*}, \beta} \mid \beta \in[0,2 \pi]\right\}$, where $\eta_{*} \in\left(-\frac{1}{\sqrt{3}}, \frac{1}{\sqrt{3}}\right)$ is 
uniquely determined by the boundary values $\eta_{+}, \eta_{-}$. The main purpose of this paper is to generalize this result to all values of $\eta_{+}, \eta_{-} \in\left(-\frac{1}{\sqrt{3}}, \frac{1}{\sqrt{3}}\right)$.

For our analysis to work, it will be necessary to make assumptions on the behavior of the solutions not only at infinity, but also in the transition region connecting the two limiting patterns. In particular, we shall assume that the solutions stay locally close to the family of steady states $\left\{U_{\eta, \beta}|| \eta \mid \leq 1, \beta \in \mathbb{R}\right\}$. Writing $u=r \mathrm{e}^{\mathrm{i} \varphi}$, this implies that the amplitude $r$ is slaved to the phase derivative $\widehat{\eta}=\partial_{x} \varphi$ in such a way that $r^{2}+\widehat{\eta}^{2} \approx 1$ for all times. In fact, we shall construct a class of solutions for which $r^{2}+\widehat{\eta}^{2}=1+\mathcal{O}(1 / t)$ as $t \rightarrow+\infty$. The behavior of these solutions will be described in a good approximation by the phase diffusion equation

$$
\partial_{t} \varphi=\partial_{x}\left[A\left(\partial_{x} \varphi\right)\right], \quad \text { where } A(\eta)=\int_{0}^{\eta} a(s) \mathrm{d} s, \quad a(s)=\frac{1-3 s^{2}}{1-s^{2}} .
$$

This equation is obtained from (1.1) by rewriting it in polar coordinates $(\varphi, r)$, and then substituting $r=\sqrt{1-\left(\partial_{x} \varphi\right)^{2}}$ into the equation for $\varphi$.

From the results in BrK92, it is apparent that the long-time dynamics of Eq.(1.3) is most naturally described in the scaling coordinates $\tau=\log t, \xi=x / \sqrt{t}$. Thus, defining $\eta(\tau, \xi)=\partial_{x} \varphi\left(\mathrm{e}^{\tau}, \mathrm{e}^{\tau / 2} \xi\right)$, the equation (1.3) is transformed into

$$
\dot{\eta}=[A(\eta)]^{\prime \prime}+\frac{\xi}{2} \eta^{\prime}, \quad \text { where }(\dot{)})=\partial_{\tau}() \text { and }()^{\prime}=\partial_{\xi}(),
$$

and the boundary conditions (1.2) become

$$
\lim _{\xi \rightarrow-\infty} \eta(\tau, \xi)=\eta_{-}, \quad \lim _{\xi \rightarrow+\infty} \eta(\tau, \xi)=\eta_{+}
$$

In Section 3 below we show that, for all $\eta_{+}, \eta_{-} \in\left(-\frac{1}{\sqrt{3}}, \frac{1}{\sqrt{3}}\right)$, Eq.(1.4) has a unique steady state $\widetilde{\eta}(\xi)$ satisfying the boundary conditions (1.5). This profile is a monotone function of $\xi$ and approaches its limits $\eta_{ \pm}$faster than any exponential. If $\left|\eta_{ \pm}\right| \ll 1$, these results were already obtained in BrK92 using a perturbation argument based on the implicit function theorem. In this case, one has the approximate expression

$$
\widetilde{\eta}(\xi) \approx \eta_{-}+\frac{\eta_{+}-\eta_{-}}{\sqrt{4 \pi}} \int_{-\infty}^{\xi} \mathrm{e}^{-z^{2} / 4} \mathrm{~d} z .
$$

Our construction is nonperturbative and relies on the monotonicity properties of the differential operator in the right-hand side of (1.4). At the same time, our monotonicity argument shows that $\widetilde{\eta}$ is a stable equilibrium point of (1.4), in the sense that any solution $\eta(\tau, \xi)$ which is sufficiently close to $\widetilde{\eta}$ in the $\mathrm{H}^{2}$ norm converges exponentially to $\widetilde{\eta}$ as $\tau \rightarrow+\infty$. Returning to the original variables, this implies that $\varphi(t, x) \approx \sqrt{t} \tilde{N}(x / \sqrt{t})$ as $t \rightarrow+\infty$, where $\widetilde{N}(\xi)=\eta_{-} \xi+\int_{-\infty}^{\xi}\left[\widetilde{\eta}(s)-\eta_{-}\right] \mathrm{d} s$.

We are now able to state our main result in a precise way. Let $\eta_{+}, \eta_{-} \in\left(-\frac{1}{\sqrt{3}}, \frac{1}{\sqrt{3}}\right)$, $\eta_{+} \neq \eta_{-}$, and assume that $\beta_{+}=\beta_{-}=0$. For $t>0$, we define the time-dependent profile

$$
\widetilde{U}(t, x)=\sqrt{1-\widetilde{\eta}(x / \sqrt{t})^{2}} \mathrm{e}^{\mathrm{i} \sqrt{t} \tilde{N}(x / \sqrt{t})},
$$

where $\widetilde{\eta}$ and $\widetilde{N}$ are constructed above. We consider initial data $u_{0} \in \mathrm{H}_{\text {loc }}^{2}(\mathbb{R})$ such that $\left\|u_{0}(\cdot)-\widetilde{U}\left(t_{0}, \cdot\right)\right\|_{\mathrm{H}^{2}} \leq \varepsilon$ for $\varepsilon>0$ sufficiently small and $t_{0}>0$ sufficiently large. Then 
Theorem 1.2 below states that the solution $u(t, x)$ of (1.1) with initial condition $u(0, \cdot)=$ $u_{0}$ satisfies

$$
\left\|u(t, \cdot)-\widetilde{U}\left(t_{0}+t, \cdot\right)\right\|_{L^{\infty}}=\mathcal{O}\left(t^{-\nu / 4}\right) \quad \text { as } t \rightarrow+\infty,
$$

for any $\nu \in(0,1)$. In particular, setting $\eta_{*}=\widetilde{\eta}(0)$ and $\varphi_{*}=\widetilde{N}(0) \neq 0$, we have for all $x_{0}>0$ :

$$
\sup _{|x| \leq x_{0}}\left|u(t, x)-U_{\eta_{*}, \varphi_{*} \sqrt{t}}(x)\right|=\mathcal{O}\left(t^{-\nu / 4}\right) \quad \text { as } t \rightarrow+\infty,
$$

see Corollary 1.3 .

This result shows that the solution $u(t, \cdot)$ converges uniformly on compact sets to the circle of steady states $\mathbf{S}_{*}=\left\{U_{\eta_{*}, \beta} \mid \beta \in[0,2 \pi]\right\}$. Following [MiS95], we can also study the convergence in the weighted norm $\mathrm{H}_{w}^{1}$ and the uniformly local norm $\mathrm{H}_{\mathrm{lu}}^{1}$ (see (4.2) below for the definitions). We find

$$
\lim _{t \rightarrow \infty} \operatorname{dist}_{\mathrm{H}_{w}^{1}}\left(u(t, \cdot), \mathbf{S}_{*}\right)=0, \quad \text { and } \liminf _{t \rightarrow \infty} \operatorname{dist}_{\mathrm{H}_{\mathrm{lu}}^{1}}\left(u(t, \cdot), \mathbf{S}_{*}\right)>0 .
$$

Indeed, in the $\mathrm{H}_{w}^{1}$ topology the solution $u(t, \cdot)$ approaches $\mathbf{S}_{*}$ sliding along the circle with nonzero speed $\varphi_{*} t^{-1 / 2}$, hence the $\omega$-limit set of $u(t, \cdot)$ is equal to $\mathbf{S}_{*}$. In the $\mathrm{H}_{\mathrm{lu}}^{1}$ topology the $\omega$-limit set of $u(t, \cdot)$ is empty.

The main ingredient in the proof of our convergence results is the use of the nonlinear change of variables defined by $u=r \mathrm{e}^{\mathrm{i} \varphi}$ and

$$
\psi(\tau, \xi)=\mathrm{e}^{-\tau / 2} \varphi\left(\mathrm{e}^{\tau}, \mathrm{e}^{\tau / 2} \xi\right)-\widetilde{N}(\xi), \quad s(\tau, \xi)=\log \left(\frac{r\left(\mathrm{e}^{\tau}, \mathrm{e}^{\tau / 2} \xi\right)^{2}}{1-\left[\partial_{x} \varphi\left(\mathrm{e}^{\tau}, \mathrm{e}^{\tau / 2} \xi\right)\right]^{2}}\right) .
$$

Remark that $s=0$ is equivalent to $r^{2}+\left(\partial_{x} \varphi\right)^{2}=1$, hence $s$ measures the slaving of the amplitude to the phase derivative. Note also that $s$ has the same regularity as $\psi^{\prime}=\partial_{\xi} \psi$. The new functions $(\psi, s)$ are solutions of a quasilinear nonautonomous parabolic system given by (2.12) below. In Section 5, we show that this system is locally well-posed in the space $\mathrm{H}^{2}(\mathbb{R}) \times \mathrm{H}^{1}(\mathbb{R})$. In Section 6 , we prove using various energy estimates that the small solutions $(\psi, s)$ of (2.12) satisfy

$$
\|\psi(\tau)\|_{\mathrm{H}^{2}(\mathbb{R})}=\mathcal{O}\left(\mathrm{e}^{-\gamma \tau}\right), \quad\|s(\tau)\|_{\mathrm{H}^{1}(\mathbb{R})}=\mathcal{O}\left(\mathrm{e}^{-\tau}\right), \quad \tau \rightarrow+\infty,
$$

for all $\gamma \in\left(0, \frac{3}{4}\right)$, see Theorem 4.1. Returning to the original variables, this implies the convergence result (1.7).

We conclude this section with a few comments on possible generalizations. Without any additional difficulty, we can extend our results to cover the equation

$$
\partial_{t} u=\partial_{x}^{2} u+h\left(|u|^{2}\right) u,
$$

where $h:[0, \infty) \rightarrow \mathbb{R}$ is a smooth, decreasing function such that $h(0)>0$ and $h(s)<0$ for sufficiently large $s$. This equation has stationary sates $U_{\eta, \beta}=H\left(\eta^{2}\right)^{1 / 2} \mathrm{e}^{\mathrm{i}(\eta x+\beta)}$, where $|\eta| \leq$ $h(0)$ and $H=h^{-1}$. The Eckhaus stability criterion takes the form $H\left(\eta^{2}\right)+2 \eta^{2} H^{\prime}\left(\eta^{2}\right)>0$, and thus is satisfied for all $\eta$ in a symmetric interval $\left(-\eta_{0}, \eta_{0}\right)$. The phase diffusion equation (1.3) becomes

$$
\partial_{t} \varphi=\partial_{x}\left[A\left(\partial_{x} \varphi\right)\right], \quad \text { where } A(\eta)=\int_{0}^{\eta}\left[1+2 s^{2} H^{\prime}\left(s^{2}\right) / H\left(s^{2}\right)\right] \mathrm{d} s .
$$


For all $\eta_{+}, \eta_{-} \in\left(-\eta_{0}, \eta_{0}\right)$, the arguments of Section 3 show the existence and uniqueness of the steady state $\widetilde{\eta}$ satisfying (1.4), (1.5), and the stability of this profile is proved as in Sections 5 and 6 .

Finally, an interesting open problem is the diffusive mixing in parabolic equations without phase invariance, such as the Swift-Hohenberg equation

$$
\partial_{t} u=-\left(1+\partial_{x}^{2}\right)^{2} u+\varepsilon u-u^{3}, \quad \varepsilon>0 .
$$

This equation also admits (if $\varepsilon$ is sufficiently small) a continuous family of spatially periodic steady states, whose stability has been proved recently [Sch96], [EWW98]. In addition, the solutions of (1.11) are well approximated for a long (but finite) interval of time by those of (1.1), see [CoE90b, [KSM92]. Therefore, a natural question is whether the results of this paper can be extended to the higher order equation (1.11).

The rest of the paper is organized as follows. In Section 2, we reformulate our problem in terms of the scaling variables $\tau, \xi$ and the new functions $\psi, s$. In Section 3, we prove the existence of the steady profile $\widetilde{\eta}$, and give a few general results on the dynamics of the phase diffusion equation (1.4). Section 1 contains the precise statements of our convergence results in both the original and the rescaled variables. The local existence of solutions for the $(\psi, s)$ system (2.12) is proved in Section 5, and Section 6 is devoted to the proof of our main result (Theorem 4.1) using energy estimates.

\section{Acknowledgments.}

The authors are grateful to G. Raugel and G. Schneider for helpful discussions. This work was begun when A.M. visited the University of Paris XI and Th.G. the University of Hannover. Both institutions are acknowledged for their hospitality. This research was partially supported by the DFG-Schwerpunktprogramm 'Dynamische Systeme' under the grant Mi 459/2-2.

\section{Reformulation of the problem}

Let $\eta_{+}, \eta_{-} \in\left(-\frac{1}{\sqrt{3}}, \frac{1}{\sqrt{3}}\right), \beta_{+}, \beta_{-} \in \mathbb{R}$, and let $u(t, x)$ be a solution of (1.1) satisfying $u(t, x) \neq 0$ for all $t, x$. Introducing the polar coordinates $(\varphi, r)$ via $u=r \mathrm{e}^{\mathrm{i} \varphi}$, we assume that the boundary conditions (1.2) hold, namely

$$
\varphi(t, x)-\eta_{ \pm} x-\beta_{ \pm} \rightarrow 0, \quad r(t, x) \rightarrow \sqrt{1-\eta_{ \pm}^{2}}, \quad \text { as } x \rightarrow \pm \infty .
$$

We suppose from now on that $\eta_{+} \neq \eta_{-}$, and refer to the end of this section for a comment on the simpler case $\eta_{+}=\eta_{-}$. We first observe that, due to the translation and phase symmetries of the Ginzburg-Landau equation (1.1), there is no loss of generality in assuming $\beta_{+}=\beta_{-}=0$. Indeed, this amounts to replacing $u(t, x)$ with $u\left(t, x-x_{0}\right) \mathrm{e}^{-\mathrm{i} \varphi_{0}}$, where

$$
x_{0}=\frac{\beta_{+}-\beta_{-}}{\eta_{+}-\eta_{-}}, \quad \varphi_{0}=\frac{\eta_{+} \beta_{-}-\eta_{-} \beta_{+}}{\eta_{+}-\eta_{-}} .
$$

Assuming thus $\beta_{ \pm}=0$, we obtain for $(\varphi, r)$ the system

$$
\partial_{t} \varphi=\partial_{x}^{2} \varphi+2 \frac{\partial_{x} r}{r} \partial_{x} \varphi, \quad \partial_{t} r=\partial_{x}^{2} r+r\left(1-r^{2}-\left[\partial_{x} \varphi\right]^{2}\right),
$$


together with the boundary conditions $\varphi(t, x)-\eta_{ \pm} x \rightarrow 0, r(t, x) \rightarrow \sqrt{1-\eta_{ \pm}^{2}}$ as $x \rightarrow \pm \infty$. For later use, we also introduce the local wave-vector $\widehat{\eta}(t, x)=\partial_{x} \varphi(t, x)$, which satisfies

$$
\partial_{t} \widehat{\eta}=\partial_{x}^{2} \widehat{\eta}+2 \partial_{x}\left(\frac{\partial_{x} r}{r} \widehat{\eta}\right), \quad \widehat{\eta}(t, x) \rightarrow \eta_{ \pm} \quad \text { as } x \rightarrow \pm \infty
$$

As explained in the introduction, we shall use the scaling coordinates $\tau=\log t, \xi=$ $x / \sqrt{t}$ to investigate the long-time dynamics of the system (2.2). Defining

$$
\eta(\tau, \xi)=\widehat{\eta}\left(\mathrm{e}^{\tau}, \mathrm{e}^{\tau / 2} \xi\right), \quad \rho(\tau, \xi)=r\left(\mathrm{e}^{\tau}, \mathrm{e}^{\tau / 2} \xi\right)
$$

we obtain the rescaled system

$$
\dot{\eta}=\eta^{\prime \prime}+\frac{\xi}{2} \eta^{\prime}+2\left(\frac{\rho^{\prime} \eta}{\rho}\right)^{\prime}, \quad \dot{\rho}=\rho^{\prime \prime}+\frac{\xi}{2} \rho^{\prime}+\mathrm{e}^{\tau} \rho\left(1-\rho^{2}-\eta^{2}\right)
$$

where $(\dot{)})=\partial_{\tau}()$ and ()$^{\prime}=\partial_{\xi}()$. This nonautonomous system has an asymptotic equilibrium point $(\widetilde{\eta}, \widetilde{\rho})$ (as $\tau \rightarrow+\infty)$ defined by $\widetilde{\rho}^{2}=1-\widetilde{\eta}^{2}$ and

$$
[A(\widetilde{\eta})]^{\prime \prime}+\frac{\xi}{2} \widetilde{\eta}^{\prime}=0, \quad \text { where } A(\eta)=\int_{0}^{\eta} \frac{1-3 s^{2}}{1-s^{2}} \mathrm{~d} s
$$

In Theorem 3.1 below, we show that the differential equation (2.4) for $\widetilde{\eta}$ has a unique solution satisfying $\widetilde{\eta}(\xi) \rightarrow \eta_{ \pm}$as $\xi \rightarrow \pm \infty$. This solution is monotone in $\xi$ and approaches its limits $\eta_{ \pm}$faster than any exponential. In addition, we have

$$
\int_{\mathbb{R}}\left[\widetilde{\eta}(\xi)-\eta_{\infty}(\xi)\right] \mathrm{d} \xi=0, \quad \text { where } \eta_{\infty}(\xi)=\left\{\begin{array}{ll}
\eta_{+} & \text {if } \xi>0 \\
\eta_{-} & \text {if } \xi<0
\end{array} .\right.
$$

The main purpose of this paper is to prove that, for suitable initial data, the solution $(\eta, \rho)$ of $(2.3)$ converges to $(\widetilde{\eta}, \widetilde{\rho})$ as $\tau \rightarrow+\infty$. For the phase $\varphi(t, x)$, this will imply the long-time behavior

$$
\varphi(t, x) \approx \eta_{-} x+\sqrt{t} \int_{-\infty}^{x / \sqrt{t}}\left[\widetilde{\eta}(\xi)-\eta_{-}\right] \mathrm{d} \xi \equiv \eta_{+} x-\sqrt{t} \int_{x / \sqrt{t}}^{+\infty}\left[\widetilde{\eta}(\xi)-\eta_{+}\right] \mathrm{d} \xi
$$

since $\partial_{x} \varphi(t, x)=\widehat{\eta}(t, x) \approx \widetilde{\eta}(x / \sqrt{t})$ and $\varphi(t, x)-\eta_{ \pm} x \rightarrow 0$ as $x \rightarrow \pm \infty$ (note that the two expressions in the right-hand side of (2.6) are identical due to (2.5)). This motivates the following ansatz for $\varphi(t, x)$. Let

$$
\widetilde{N}(\xi)=\eta_{-} \xi+\int_{-\infty}^{\xi}\left[\widetilde{\eta}(s)-\eta_{-}\right] \mathrm{d} s \equiv \eta_{+} \xi-\int_{\xi}^{+\infty}\left[\widetilde{\eta}(s)-\eta_{+}\right] \mathrm{d} s .
$$

We define

$$
\psi(\tau, \xi)=\mathrm{e}^{-\tau / 2} \varphi\left(\mathrm{e}^{\tau}, \mathrm{e}^{\tau / 2} \xi\right)-\tilde{N}(\xi), \quad \rho(\tau, \xi)=r\left(\mathrm{e}^{\tau}, \mathrm{e}^{\tau / 2} \xi\right),
$$

or equivalently

$$
\varphi(t, x)=\sqrt{t}[\widetilde{N}(x / \sqrt{t})+\psi(\log t, x / \sqrt{t})], \quad r(t, x)=\rho(\log t, x / \sqrt{t}) .
$$


Then $\psi(\tau, \xi) \rightarrow 0$ as $\xi \rightarrow \pm \infty$, and (2.2) is transformed into

$$
\begin{aligned}
\dot{\psi} & =(\tilde{N}+\psi)^{\prime \prime}+\frac{\xi}{2}(\tilde{N}+\psi)^{\prime}-\frac{1}{2}(\tilde{N}+\psi)+2 \frac{\rho^{\prime}}{\rho} \eta \\
\dot{\rho} & =\rho^{\prime \prime}+\frac{\xi}{2} \rho^{\prime}+\mathrm{e}^{\tau} \rho\left(1-\rho^{2}-\eta^{2}\right)
\end{aligned}
$$

where $\eta=(\widetilde{N}+\psi)^{\prime}=\widetilde{\eta}+\psi^{\prime}$.

By construction, we expect that the solution $(\psi, \rho)$ of $(2.10)$ converges to $(0, \widetilde{\rho})$ as $\tau \rightarrow+\infty$, where $\widetilde{\rho}^{2}=1-\widetilde{\eta}^{2}$. Assuming this to hold, we deduce from the second equation in (2.10) that $\rho(\tau, \xi)^{2}=1-\eta(\tau, \xi)^{2}+\mathcal{O}\left(\mathrm{e}^{-\tau}\right)$. This is the so-called "slaving" of the amplitude $\rho$ to the phase derivative $\eta$. To take this behavior into account, we parametrize the amplitude $\rho$ with the new variable $s$ defined by

$$
\rho=\sqrt{1-\eta^{2}} \mathrm{e}^{s / 2} \equiv \sqrt{1-\left(\widetilde{\eta}+\psi^{\prime}\right)^{2}} \mathrm{e}^{s / 2} .
$$

After some elementary calculations, we obtain the following equations for $\psi, s$

$$
\begin{aligned}
\dot{\psi} & =\left[A\left(\widetilde{\eta}+\psi^{\prime}\right)-A(\widetilde{\eta})\right]^{\prime}+\frac{\xi}{2} \psi^{\prime}-\frac{1}{2} \psi+\eta s^{\prime} \\
\dot{s} & =a_{1}(\eta) s^{\prime \prime}+a_{2}(\eta) \eta^{\prime \prime}+\frac{\xi}{2} s^{\prime}-2 \mathrm{e}^{\tau}\left(1-\eta^{2}\right)\left(\mathrm{e}^{s}-1\right)+\frac{1}{2} s^{\prime 2}+a_{3}(\eta) \eta^{\prime 2}
\end{aligned}
$$

where $\eta=\widetilde{\eta}+\psi^{\prime}$. The coefficients $a=A^{\prime}$ and $a_{1}, a_{2}, a_{3}$ are given by

$$
a(\eta)=\frac{1-3 \eta^{2}}{1-\eta^{2}}, \quad a_{1}(\eta)=\frac{1+\eta^{2}}{1-\eta^{2}}, \quad a_{2}(\eta)=\frac{-4 \eta^{3}}{\left(1-\eta^{2}\right)^{2}}, \quad a_{3}(\eta)=\frac{-2-6 \eta^{2}}{\left(1-\eta^{2}\right)^{3}}
$$

This is the final form of the problem which is the basis of our analysis in Sections 5 and 6. In particular, our main result (Theorem 4.1) will show that sufficiently small solutions $(\psi, s)$ of (2.12) satisfy the decay estimate (1.10). Remark that there is now an imbalance in the number of spatial derivatives of $\psi$ and $s$ in (2.12), since the $s$-equation contains $\eta^{\prime \prime}=\widetilde{\eta}^{\prime \prime}+\psi^{\prime \prime \prime}$, whereas only $s^{\prime}$ appears in the $\psi$-equation. This derives from the fact that $\rho$ is slaved to $\eta=\widetilde{\eta}+\psi^{\prime}$. Due to this imbalance, we also have to consider the equation for $\delta=\psi^{\prime}=\eta-\widetilde{\eta}$ which reads

$$
\dot{\delta}=[A(\widetilde{\eta}+\delta)-A(\widetilde{\eta})]^{\prime \prime}+\frac{\xi}{2} \delta^{\prime}+\left((\widetilde{\eta}+\delta) s^{\prime}\right)^{\prime} .
$$

Remark. When $\eta_{+}=\eta_{-}$, the discussion above remains valid, except for an important difference : if $\beta_{+} \neq \beta_{-}$, it is no longer possible to reduce the problem to $\beta_{ \pm}=0$ using the symmetries of the Ginzburg-Landau equation. As a consequence, the function $\psi(\tau, \xi)$ defined by (2.8) has non trivial boundary values $\psi(\tau, \pm \infty)=\beta_{ \pm}$, and $\int \delta(\tau, \xi) \mathrm{d} \xi=$ $\beta_{+}-\beta_{-} \neq 0$. It follows that $\delta(\tau, \cdot)$ converges to zero like $e^{-\tau / 2}$ as $\tau \rightarrow+\infty$, and not faster as in the previous case. Indeed, since $\widetilde{\eta}(\xi)=\eta_{+}=\eta_{-}$is now constant, the linearized equation (2.14) becomes

$$
\dot{\delta}=\widetilde{a} \delta^{\prime \prime}+\frac{\xi}{2} \delta^{\prime}+\widetilde{\eta} s^{\prime \prime}, \quad \text { where } \widetilde{a}=a(\widetilde{\eta}) .
$$

As is well-known, the largest eigenvalue of the operator $\widetilde{a} \partial_{\xi}^{2}+\frac{\xi}{2} \partial_{\xi}$ is $-1 / 2$, with eigenfunction $\omega(\xi)=(4 \pi \widetilde{a})^{-1 / 2} \exp \left(-\xi^{2} / 4 \widetilde{a}\right)$ and adjoint eigenfunction 1. Since $s(\tau, \cdot)=\mathcal{O}\left(e^{-\tau}\right)$ 
due to the slaving, we conclude that $\delta(\tau, \xi) \approx e^{-\tau / 2} m(\xi)$ as $\tau \rightarrow+\infty$, where $m(\xi)=$ $\left(\beta_{+}-\beta_{-}\right) \omega(\xi)$. Therefore, a natural ansatz for $\psi$ is $\psi(\tau, \xi)=e^{-\tau / 2} M(\xi)+\chi(\tau, \xi)$, where

$$
M(\xi)=\beta_{-}+\frac{\beta_{+}-\beta_{-}}{(4 \pi \widetilde{a})^{1 / 2}} \int_{-\infty}^{\xi} \mathrm{e}^{-y^{2} /(4 \widetilde{a})} \mathrm{d} y, \quad M^{\prime}(\xi)=m(\xi) .
$$

With this definition, the equations for $\chi, s$ are

$$
\begin{aligned}
\dot{\chi} & =\left[A\left(\widetilde{\eta}+e^{-\tau / 2} m+\chi^{\prime}\right)-A\left(\widetilde{\eta}+e^{-\tau / 2} m\right)\right]^{\prime}+\frac{\xi}{2} \chi^{\prime}-\frac{1}{2} \chi+\eta s^{\prime}+\Lambda \\
\dot{s} & =a_{1}(\eta) s^{\prime \prime}+a_{2}(\eta) \eta^{\prime \prime}+\frac{\xi}{2} s^{\prime}-2 \mathrm{e}^{\tau}\left(1-\eta^{2}\right)\left(\mathrm{e}^{s}-1\right)+\frac{1}{2} s^{2}+a_{3}(\eta) \eta^{\prime 2}
\end{aligned}
$$

where $\eta=\widetilde{\eta}+e^{-\tau / 2} m+\chi^{\prime}$ and $\Lambda(\tau, \xi)=e^{-\tau / 2}\left[a\left(\widetilde{\eta}+e^{-\tau / 2} m\right)-a(\widetilde{\eta})\right] m^{\prime}=\mathcal{O}\left(e^{-\tau}\right)$. Then, proceeding as in Section 6, one can show that $\|\chi(\tau, \cdot)\|_{\mathrm{H}^{2}}=\mathcal{O}\left(\mathrm{e}^{-\gamma \tau}\right)$ and $\|s(\tau, \cdot)\|_{\mathrm{H}^{1}}=$ $\mathcal{O}\left(\mathrm{e}^{-\tau}\right)$ as $\tau \rightarrow+\infty$, for all $\gamma \in(0,3 / 4)$.

Remark. An important features of the Ginzburg-Landau equation are its symmetry properties which are essential to our analysis in several respects. Eq. (1.1) is invariant under the phase rotations $P_{\alpha}$ and the translations in time $T_{z}$ and space $S_{y}$ defined by

$$
\begin{array}{lll}
T_{z}:(t, x, u) & \mapsto(t+z, x, u), & z \in \mathbb{R} ; \\
S_{y}:(t, x, u) \mapsto(t, x+y, u), & y \in \mathbb{R} ; \\
P_{\alpha}: \quad(t, x, u) \mapsto\left(t, x, \mathrm{e}^{\mathrm{i} \alpha} u\right), & & \alpha \in \mathbb{R} .
\end{array}
$$

For the transformed system (2.3), the rotation $P_{\alpha}$ is trivial, and the translations $T_{z}, S_{y}$ become

$$
\begin{aligned}
& T_{z}: \quad(\tau, \xi, \eta, \rho) \mapsto\left(\tau+\log \left(1+\mathrm{e}^{-\tau} z\right), \xi / \sqrt{1+\mathrm{e}^{-\tau} z}, \eta, \rho\right) ; \\
& S_{y}: \quad(\tau, \xi, \eta, \rho) \mapsto\left(\tau, \xi+\mathrm{e}^{-\tau / 2} y, \eta, \rho\right) .
\end{aligned}
$$

The transformation rules for $\psi(\tau, \xi)$ and $\delta(\tau, \xi)$ are more complicated and will not be used in the sequel.

\section{The phase diffusion equation}

In this section, we first prove the existence of a unique solution $\widetilde{\eta}(\xi)$ of $(2.4)$ satisfying the boundary conditions $\widetilde{\eta}(\xi) \rightarrow \eta_{ \pm}$as $\xi \rightarrow \pm \infty$. We next study the structure and dynamics of the phase diffusion equation (or slaved equation)

$$
\dot{\psi}=\left[A\left(\widetilde{\eta}+\psi^{\prime}\right)\right]^{\prime}+\frac{\xi}{2}\left(\widetilde{\eta}+\psi^{\prime}\right)-\frac{1}{2}(\widetilde{N}+\psi)
$$

which is obtained by setting $s=0$ in the $\psi$-equation of (2.12). Since $s(\tau, \cdot)$ decays to zero like $e^{-\tau}$ as $\tau \rightarrow+\infty$ due to the slaving, it is reasonable to expect that the long-time dynamics of the full system (2.12) is well approximated by (3.1), even though $s \equiv 0$ does not define an invariant submanifold. In addition, some structures of the full problem can be understood better on this simpler model. We begin with the existence of the steady state: 


\section{Theorem 3.1}

(a) For each $\eta_{+}, \eta_{-} \in\left(-\frac{1}{\sqrt{3}}, \frac{1}{\sqrt{3}}\right)$ there exists a unique $\widetilde{\eta} \in \mathcal{C}^{2}\left(\mathbb{R},\left(-\frac{1}{\sqrt{3}}, \frac{1}{\sqrt{3}}\right)\right)$ solving

$$
[A(\widetilde{\eta})]^{\prime \prime}+\frac{\xi}{2} \widetilde{\eta}^{\prime}=0 \text { on } \mathbb{R}, \quad \text { and } \quad \widetilde{\eta}(\xi) \rightarrow \eta_{ \pm} \text {as } \xi \rightarrow \pm \infty,
$$

where $A(\eta)$ is defined in (2.4).

(b) The solution $\widetilde{\eta}$ is constant if $\eta_{+}=\eta_{-}$and strictly monotone otherwise. In addition, for each $C>0$ we have $\sup \left\{\mathrm{e}^{C|\xi|}\left|\widetilde{\eta}^{\prime}(\xi)\right| \mid \xi \in \mathbb{R}\right\}<\infty$.

(c) The function $\widetilde{\eta}$ satisfies

$$
\int_{\mathbb{R}}\left[\widetilde{\eta}(\xi)-\eta_{\infty}(\xi)\right] \mathrm{d} \xi=0 \quad \text { and } \quad \int_{\mathbb{R}} \xi\left[\widetilde{\eta}(\xi)-\eta_{\infty}(\xi)\right] \mathrm{d} \xi=A\left(\eta_{-}\right)-A\left(\eta_{+}\right),
$$

where $\eta_{\infty}$ is defined in (2.5). Setting $\varphi_{*}=\int_{-\infty}^{0}\left[\widetilde{\eta}(\xi)-\eta_{-}\right] \mathrm{d} \xi=\int_{0}^{+\infty}\left[\eta_{+}-\widetilde{\eta}(\xi)\right] \mathrm{d} \xi$, we have $\operatorname{sign}\left(\varphi_{*}\right)=\operatorname{sign}\left(\eta_{+}-\eta_{-}\right)$, and hence $\varphi_{*} \neq 0$ if $\eta_{+} \neq \eta_{-}$.

Proof: Let $\eta_{+}, \eta_{-} \in\left(-\frac{1}{\sqrt{3}}, \frac{1}{\sqrt{3}}\right)$. The existence of the steady profile $\widetilde{\eta}$ will be obtained using the theory of (nonlinear) monotone operators. First, choosing $\varepsilon>0$ sufficiently small, we modify $a(\eta)=\left(1-3 \eta^{2}\right) /\left(1-\eta^{2}\right)$ outside the interval containing $\eta_{+}, \eta_{-}$in such a way that the resulting function (still denoted by $a$ ) is $\mathcal{C}^{\infty}$ and satisfies $1 \geq a(\eta) \geq$ $\varepsilon>0$ for all $\eta \in \mathbb{R}$. Accordingly, the modified primitive $A(\eta)=\int_{0}^{\eta} a(y) \mathrm{d} y$ verifies $\left(\eta_{1}-\eta_{2}\right)\left(A\left(\eta_{1}\right)-A\left(\eta_{2}\right)\right) \geq \varepsilon\left(\eta_{1}-\eta_{2}\right)^{2}$ for all $\eta_{1}, \eta_{2} \in \mathbb{R}$. Next, we choose a smooth, monotone function $n: \mathbb{R} \rightarrow \mathbb{R}$ such that

$$
\left|n^{\prime}(\xi)\right| \leq C \mathrm{e}^{-|\xi|}, \quad\left|n(\xi)-\eta_{\infty}(\xi)\right| \leq C \mathrm{e}^{-|\xi|}, \quad \int_{\mathbb{R}}\left[n(\xi)-\eta_{\infty}(\xi)\right] \mathrm{d} \xi=0,
$$

for some $C>0$, where $\eta_{\infty}$ is given by (2.5). In analogy with (2.7), we set

$$
N(\xi)=\eta_{-} \xi+\int_{-\infty}^{\xi}\left[n(s)-\eta_{-}\right] \mathrm{d} s \equiv \eta_{+} \xi-\int_{\xi}^{+\infty}\left[n(s)-\eta_{+}\right] \mathrm{d} s,
$$

and we observe that $|N(\xi)-\xi n(\xi)| \leq C \mathrm{e}^{-|\xi|}$. Finally, we define the operators $B_{1}$ : $\mathrm{H}^{1}(\mathbb{R}) \rightarrow \mathrm{H}^{-1}(\mathbb{R})$ and $B_{2}: D(B) \subset \mathrm{H}^{1}(\mathbb{R}) \rightarrow \mathrm{H}^{-1}(\mathbb{R})$ by

$$
\begin{aligned}
D(B) & =\left\{\psi \in \mathrm{H}^{1}(\mathbb{R}) \mid \xi \psi^{\prime} \in \mathrm{H}^{-1}(\mathbb{R})\right\}, \\
B_{1}(\psi) & =-\left[A\left(n+\psi^{\prime}\right)\right]^{\prime}+\frac{1}{2} \psi, \\
B_{2} \psi & =-\frac{\xi}{2} \psi^{\prime} .
\end{aligned}
$$

The program is to show that the nonlinear equation

$$
B_{1}(\psi)+B_{2} \psi=\frac{1}{2}[\xi n(\xi)-N(\xi)] \in \mathrm{H}^{-1}(\mathbb{R})
$$

has a unique solution $\widetilde{\psi} \in D(B) \subset \mathrm{H}^{1}(\mathbb{R})$. Then, $\widetilde{\eta}=n+\widetilde{\psi}^{\prime}$ will be the desired solution of (3.2).

To prove the existence and uniqueness of the solution of (3.4), we first observe that $B_{1}, B_{2}$ are coercive (or strongly monotone) operators, with

$$
\begin{aligned}
\left\langle B_{1}\left(\psi_{1}\right)-B_{1}\left(\psi_{2}\right), \psi_{1}-\psi_{2}\right\rangle_{\mathrm{H}^{-1} \times \mathrm{H}^{1}} & \geq \varepsilon\left\|\psi_{1}^{\prime}-\psi_{2}^{\prime}\right\|_{\mathrm{L}^{2}}^{2}+\frac{1}{2}\left\|\psi_{1}-\psi_{2}\right\|_{\mathrm{L}^{2}}^{2} \forall \psi_{1}, \psi_{2} \in \mathrm{H}^{1}, \\
\left\langle B_{2}\left(\psi_{1}-\psi_{2}\right), \psi_{1}-\psi_{2}\right\rangle_{\mathrm{H}^{-1} \times \mathrm{H}^{1}} & =\frac{1}{4}\left\|\psi_{1}-\psi_{2}\right\|_{\mathrm{L}^{2}}^{2} \forall \psi_{1}, \psi_{2} \in D(B) .
\end{aligned}
$$


Clearly the nonlinear operator $B_{1}$ is maximal monotone as it is continuous (from $\mathrm{H}^{1}$ into its dual $\mathrm{H}^{-1}$ ) and monotone, see Zei90 Proposition 32.7. On the other hand, the linear operator $B_{2}$ is densely defined, and a direct calculation shows that the adjoint operator $B_{2}^{*}$ satisfies $D\left(B_{2}^{*}\right)=D(B)$ and $B_{2}^{*}=-B_{2}+\frac{1}{2}$. Thus $B_{2}$ is closed, $B_{2}^{*}$ is monotone, hence $B_{2}$ is maximal monotone by [Zei90 Theorem 32.L. Since int $D\left(B_{1}\right) \cap D\left(B_{2}\right)=$ $\mathrm{H}^{1}(\mathbb{R}) \cap D(B)=D(B) \neq \emptyset$, it follows from the "sum theorem" (see [Zei90] Theorem 32.I) that $B=B_{1}+B_{2}: D(B) \rightarrow \mathrm{H}^{-1}(\mathbb{R})$ is maximal monotone. As $B$ is also coercive, we conclude that $B$ is one-to-one from $D(B)$ onto $\mathrm{H}^{-1}(\mathbb{R})$ ([Zei90] Theorem 32.G), hence there exists a unique $\widetilde{\psi} \in D(B) \subset \mathrm{H}^{1}(\mathbb{R})$ such that (3.4) holds.

It remains to show that $\widetilde{\psi}$ is smooth, and that $\widetilde{\eta}=n+\widetilde{\psi}^{\prime}$ remains in the interval between $\eta_{+}$and $\eta_{-}$where no modification of $a$ was needed. Since $a(\eta)=A^{\prime}(\eta) \geq \varepsilon>0$, classical regularity theory for ordinary differential equations applied to (3.4) shows that $\widetilde{\psi}$ is as smooth as the coefficients in the equation, namely $\widetilde{\psi} \in \mathcal{C}^{\infty}(\mathbb{R})$. Hence $\widetilde{\eta}=n+\widetilde{\psi}^{\prime}$ solves

$$
[A(\widetilde{\eta})]^{\prime \prime}+\frac{\xi}{2} \widetilde{\eta}^{\prime}=0, \quad \text { or equivalently }\left(a(\widetilde{\eta}) \widetilde{\eta}^{\prime}\right)^{\prime}=-\frac{\xi}{2 a(\widetilde{\eta})}\left(a(\widetilde{\eta}) \widetilde{\eta}^{\prime}\right) .
$$

Integrating this equation, we obtain for any $\xi_{0} \in \mathbb{R}$

$$
\widetilde{\eta}^{\prime}(\xi)=\frac{a\left(\widetilde{\eta}\left(\xi_{0}\right)\right) \widetilde{\eta}^{\prime}\left(\xi_{0}\right)}{a(\widetilde{\eta}(\xi))} \exp \left(-\int_{\xi_{0}}^{\xi} \frac{y}{2 a(\widetilde{\eta}(y))} \mathrm{d} y\right), \quad \xi \in \mathbb{R} .
$$

From this representation, it is obvious that $\widetilde{\eta}^{\prime}\left(\xi_{0}\right)$ cannot vanish unless $\widetilde{\eta}$ is identically constant, hence $\widetilde{\eta}$ is a monotone function of $\xi \in \mathbb{R}$. In addition, since $a(\widetilde{\eta}) \geq \varepsilon>0$, it follows from (3.5) that $\widetilde{\eta}^{\prime}$ converges to 0 faster than any exponential as $\xi \rightarrow \pm \infty$. Therefore, $\widetilde{\eta}$ converge to some limits as $\xi \rightarrow \pm \infty$, and these limits must be the boundary value $\eta_{ \pm}$prescribed by $n$, since $\widetilde{\eta}-n=\widetilde{\psi}^{\prime} \in \mathrm{L}^{2}(\mathbb{R})$. Thus, parts (a) and (b) are established. The relations in (c) follow from (3.2) by partial integration, taking care of the jump of $\eta_{\infty}$ at $\xi=0$. Finally, $\operatorname{sign}\left(\varphi_{*}\right)=\operatorname{sign}\left(\eta_{+}-\eta_{-}\right)$since $\widetilde{\eta}$ is strictly monotone if $\eta_{+} \neq \eta_{-}$.

Strictly speaking, the remainder of this section is not needed for the proof of the main results as given in Section 1 . However, the statements and proofs given here for the slaved problem (3.1) help to understand the analysis of the more difficult full problem (2.12). Moreover, the arguments here show that our decay results are in a certain sense optimal.

In the above theorem we used the fact that the steady part of (3.1) has a monotone structure. This in fact implies that the dynamics of (3.1) is rather trivial: all solutions are contracted with prescribed rate towards the unique steady state $\psi=0$. Let $\psi_{1}, \psi_{2}$ be classical solutions of (3.1) in $\mathrm{H}^{2}(\mathbb{R})$ satisfying $\widetilde{\eta}(\xi)+\psi_{j}^{\prime}(\tau, \xi) \in\left(-\frac{1}{\sqrt{3}}, \frac{1}{\sqrt{3}}\right)$ for all $\tau \geq 0$ and $\xi \in \mathbb{R}$. Then

$$
\left\|\psi_{1}(\tau, \cdot)-\psi_{2}(\tau, \cdot)\right\|_{\mathrm{L}^{2}(\mathbb{R})} \leq \mathrm{e}^{-3 \tau / 4}\left\|\psi_{1}(0, \cdot)-\psi_{2}(0, \cdot)\right\|_{\mathrm{L}^{2}(\mathbb{R})},
$$

for all $\tau \geq 0$. This follows from the energy estimate

$$
\begin{aligned}
\frac{1}{2} \frac{d}{d t} \int_{\mathbb{R}}\left(\psi_{1}-\psi_{2}\right)^{2} \mathrm{~d} \xi= & \int_{\mathbb{R}}\left(\psi_{1}-\psi_{2}\right)\left[A\left(\widetilde{\eta}+\psi_{1}^{\prime}\right)^{\prime}-A\left(\widetilde{\eta}+\psi_{2}^{\prime}\right)^{\prime}\right] \mathrm{d} \xi \\
& +\int_{\mathbb{R}}\left(\psi_{1}-\psi_{2}\right)\left[\frac{\xi}{2}\left(\psi_{1}^{\prime}-\psi_{2}^{\prime}\right)-\frac{1}{2}\left(\psi_{1}-\psi_{2}\right)\right] \mathrm{d} \xi \\
= & -\int_{\mathbb{R}}\left(\psi_{1}^{\prime}-\psi_{2}^{\prime}\right)\left[A\left(\widetilde{\eta}+\psi_{1}^{\prime}\right)-A\left(\widetilde{\eta}+\psi_{2}^{\prime}\right)\right] \mathrm{d} \xi-\frac{3}{4} \int_{\mathbb{R}}\left(\psi_{1}-\psi_{2}\right)^{2} \mathrm{~d} \xi
\end{aligned}
$$


The first integral in the last row is nonnegative since $A$ is monotone, namely, $A^{\prime}(\eta)=$ $a(\eta)>0$ implying $\left(\eta_{1}-\eta_{2}\right)\left(A\left(\eta_{1}\right)-A\left(\eta_{2}\right)\right) \geq 0$. Applying Gronwall's inequality, we obtain (3.6).

We now consider the linearization of the phase diffusion equation (3.1) at the origin, and we discuss the spectral properties of the corresponding differential operator $\mathbf{L}: \psi \mapsto$ $\left[a(\widetilde{\eta}) \psi^{\prime}\right]^{\prime}+\frac{\xi}{2} \psi^{\prime}-\frac{1}{2} \psi$. According to $(3.6)$, the spectrum of $\mathbf{L}$ in $\mathrm{L}^{2}(\mathbb{R})$ is contained in the left half-plane $\Omega_{0}=\{z \in \mathbb{C} \mid \operatorname{Re}(z) \leq-3 / 4\}$. In fact, our next result will show that $\sigma(\mathbf{L})=\Omega_{0}$ in $\mathrm{L}^{2}(\mathbb{R})$, so that the estimate (3.6) cannot be improved. However, the upper bound of the spectrum depends on the function space, and can be shifted to the left if we impose a faster decay at infinity. To see this, we introduce for $\ell \geq 0$ the weighted space $X^{\ell}=\mathrm{L}^{2}\left(\mathbb{R},\left(1+\xi^{2}\right)^{\ell} \mathrm{d} \xi\right)$ equipped with the scalar product $\langle\psi, \varphi\rangle=\int_{\mathbb{R}} \psi \varphi\left(1+\xi^{2}\right)^{\ell} \mathrm{d} \xi$. Then the following holds:

\section{Proposition 3.2}

Let $\ell \geq 0$ and let $\mathbf{L}: D(\mathbf{L}) \subset X^{\ell} \rightarrow X^{\ell}$ be the linear operator defined by $D(\mathbf{L})=\{\psi \in$ $\left.X^{\ell} \mid \psi^{\prime \prime} \in X^{\ell}, \xi \psi^{\prime} \in X^{\ell}\right\}$ and $\mathbf{L} \psi=\left(a(\widetilde{\eta}) \psi^{\prime}\right)^{\prime}+\frac{\xi}{2} \psi^{\prime}-\frac{1}{2} \psi$ for all $\psi \in D(\mathbf{L})$. Then there

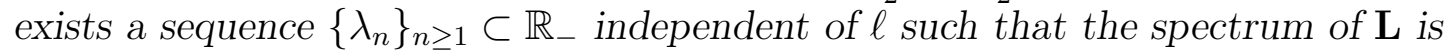

$$
\sigma(\mathbf{L})=\left\{\lambda_{n} \mid n \geq 1\right\} \cup\{z \in \mathbb{C} \mid \operatorname{Re}(z) \leq-3 / 4-\ell / 2\} .
$$

In addition, $\lambda_{1}=-1, \lambda_{n+1}<\lambda_{n}$ for all $n \geq 1, \lambda_{n} \rightarrow-\infty$ as $n \rightarrow+\infty$, and $\lambda_{n}$ is a simple (isolated) eigenvalue of $\mathbf{L}$ for all $n$ such that $\lambda_{n}>-3 / 4-\ell / 2$.

\section{Remarks.}

1) In the case where $\eta_{+}=\eta_{-}=\widetilde{\eta}$, it is known that $\lambda_{n}=-(n+1) / 2$ for all $n \geq 1$, see for instance GR97. The remarkable fact that $\lambda_{1}=-1$ is the largest isolated eigenvalue of $\mathbf{L}$ for all $\eta_{+}, \eta_{-}$originates in the translation invariance in time of the Ginzburg-Landau equation (1.1), see the discussion at the end of this section.

2) Proposition 3.2 gives the upper bound $\max \{-1,-3 / 4-\ell / 2\}$ on the real part of the spectrum of $\mathbf{L}$ in $X^{\ell}$. Thus, even for rapidly decreasing initial data, the solutions of (3.1) do not converge to zero faster than $e^{-\tau}$ as $\tau \rightarrow+\infty$.

Proof: Throughout the proof, we denote by $\Omega_{\ell}$ the half-plane $\{z \in \mathbb{C} \mid \operatorname{Re}(z) \leq-3 / 4-$ $\ell / 2\}$. Let $b(\xi)=a(\widetilde{\eta}(\xi))$, and let $\gamma(\xi)$ be the solution of the differential equation $4 b(\xi) \gamma^{\prime}(\xi)+\xi \gamma(\xi)=0$ with initial condition $\gamma(0)=1$. By Theorem 3.1, $b(\xi)$ converges (faster than any exponential) to positive limits $b_{ \pm}$as $\xi \rightarrow \pm \infty$, hence $\gamma(\xi)=$ $\mathcal{O}\left(\exp \left(-\xi^{2} / 8 b_{ \pm}\right)\right)$as $\xi \rightarrow \pm \infty$.

For all $\lambda \in \mathbb{C}$, it is easy to verify (using asymptotic expansions) that the linear ODE $\mathbf{L} \psi=\lambda \psi$ has two independent solutions $\psi_{1}^{+}, \psi_{2}^{+}$satisfying

$$
\psi_{1}^{+}(\xi)=\xi^{1+2 \lambda}\left(1+\mathcal{O}\left(\xi^{-2}\right)\right), \quad \psi_{2}^{+}(\xi)=\xi^{-2(\lambda+1)} \gamma(\xi)^{2}\left(1+\mathcal{O}\left(\xi^{-2}\right)\right)
$$

as $\xi \rightarrow+\infty$. Similarly, there exist independent solutions $\psi_{1}^{-}, \psi_{2}^{-}$such that

$$
\psi_{1}^{-}(\xi)=|\xi|^{1+2 \lambda}\left(1+\mathcal{O}\left(|\xi|^{-2}\right)\right), \quad \psi_{2}^{-}(\xi)=|\xi|^{-2(\lambda+1)} \gamma(\xi)^{2}\left(1+\mathcal{O}\left(|\xi|^{-2}\right)\right)
$$

as $\xi \rightarrow-\infty$. Therefore, if $\operatorname{Re}(\lambda)<-3 / 4-\ell / 2$, any solution $\psi$ to $\mathbf{L} \psi=\lambda \psi$ belongs to $X^{\ell}$, so that $\lambda$ is a double eigenvalue of $\mathbf{L}$. This shows that $\sigma(\mathbf{L}) \supset \Omega_{\ell}$. 
On the other hand, if $\lambda$ is any eigenvalue of $\mathbf{L}$ with $\operatorname{Re}(\lambda)>-3 / 4-\ell / 2$, then (by the same argument) $\lambda$ is simple and the corresponding eigenvector $\psi$ decays like $|\xi|^{-2(\lambda+1)} \gamma(\xi)^{2}$ as $\xi \rightarrow \pm \infty$. Thus, setting $\varphi(\xi)=\gamma(\xi)^{-1} \psi(\xi)$, we see that $\varphi \in \mathrm{H}^{2}(\mathbb{R})$ is a solution to $\Lambda \varphi=\lambda \varphi$, where

$$
\Lambda \varphi=\left(b \varphi^{\prime}\right)^{\prime}-V \varphi, \quad \text { and } V(\xi)=\frac{3}{4}+\frac{\xi^{2}}{16 b(\xi)} .
$$

Since $b(\xi) \rightarrow b_{ \pm}$and $V(\xi) \rightarrow+\infty$ as $\xi \rightarrow \pm \infty$, it is well-known (see for instance [CL55], chapter 9) that $\Lambda$ is self-adjoint in $\mathrm{L}^{2}(\mathbb{R})$, and that its spectrum consists of a sequence $\left\{\lambda_{n}\right\}_{n \geq 1}$ of real, simple eigenvalues satisfying $\lambda_{n+1}<\lambda_{n}$ and $\lambda_{n} \rightarrow-\infty$ as $n \rightarrow+\infty$. Moreover, the eigenfunction $\varphi_{n}$ associated with $\lambda_{n}$ has exactly $n$ zeros in $\mathbb{R}$. This proves that, for all $n \geq 1, \lambda_{n}$ is an eigenvalue of $\mathbf{L}$ with eigenfunction $\psi_{n}=\gamma \varphi_{n}$, and that the sequence $\left\{\lambda_{n}\right\}_{n \geq 1}$ exhausts the discrete spectrum of $\mathbf{L}$ in the half-plane $\Omega_{\ell}^{c}$. Now, a direct calculation shows that $\mathbf{L} \hat{\psi}=-\hat{\psi}$, where $\hat{\psi}(\xi)=2 b(\xi) \widetilde{\eta}^{\prime}(\xi)$ if $\eta_{+} \neq \eta_{-}$and $\hat{\psi}(\xi)=\exp \left(-\xi^{2} / 4 b\right)$ if $\eta_{+}=\eta_{-}$. Since $\hat{\psi}$ has no zero in $\mathbb{R}$, it follows that $\lambda_{1}=-1$ and $\psi_{0}=\hat{\psi}$.

It remains to verify that $\mathbf{L}$ has no essential spectrum in $\Omega_{\ell}^{c}$ (in other words, if $\lambda \in$ $\sigma(\mathbf{L}) \cap \Omega_{\ell}^{c}$, then $\lambda$ is an isolated eigenvalue of $\mathbf{L}$ with finite algebraic multiplicity.) To prove this, we use the change of variables defined by $y=B(\xi)=\int_{0}^{\xi} b(z)^{-1 / 2} \mathrm{~d} z, \psi(\xi)=\chi(B(\xi))$. Then $(\mathbf{L} \psi)(\xi)=(\mathcal{L} \chi)(B(\xi))$, where

$$
(\mathcal{L} \chi)(y)=\chi^{\prime \prime}(y)+\frac{y}{2} \chi^{\prime}(y)-\frac{1}{2} \chi(y)+D(y) \chi^{\prime}(y) \equiv\left(\mathbf{L}_{0} \chi\right)(y)+D(y) \chi^{\prime}(y),
$$

and

$$
D(y)=\left.\left(\frac{1}{2} b^{\prime}(\xi)+\xi b(\xi)^{-1 / 2}-B(\xi)\right)\right|_{\xi=B^{-1}(y)} .
$$

From [GR97, we know that $\sigma_{\text {ess }}\left(\mathbf{L}_{0}\right)=\Omega_{\ell}$ in $X^{\ell}$. On the other hand, it follows from the Rellich criterion ( RS72], theorem XIII.65) that the operator $\partial_{y} \mathbf{L}_{0}^{-1}$ is compact in $X^{\ell}$. Indeed, there exists a constant $C>0$ such that, for all $\psi \in X^{\ell}$ with $\|\psi\|_{X^{\ell}} \leq 1$, the function $\chi=\partial_{y}\left(\mathbf{L}_{0}^{-1} \psi\right)$ satisfies $\|\chi\|_{X^{\ell}} \leq C,\|y \chi\|_{X^{\ell}} \leq C,\left\|\chi^{\prime}\right\|_{X^{\ell}} \leq C$. Since the function $D(y)$ is bounded, we conclude that $\mathcal{L}$ is a relatively compact perturbation of $\mathbf{L}_{0}$. Now, it follows from a classical theorem ([He81], theorem A.1, p. 136) that either $\sigma_{\text {ess }}(\mathcal{L})=\sigma_{\text {ess }}\left(\mathbf{L}_{0}\right)=\Omega_{\ell}$, or the half-plane $\Omega_{\ell}^{c}$ is filled with eigenvalues of $\mathcal{L}$. The second possibility is of course excluded, hence we have shown that $\sigma_{\text {ess }}(\mathbf{L}) \equiv \sigma_{\text {ess }}(\mathcal{L})=\Omega_{\ell}$.

Finally, we show that the eigenvalue $\lambda_{1}=-1$ of $\mathbf{L}$ is related to the symmetry properties (2.16) of the Ginzburg-Landau equation (1.1). It is advantageous to discuss these symmetries for the slaved equation for $\eta$ given by

$$
\dot{\eta}=[A(\eta)]^{\prime \prime}+\frac{\xi}{2} \eta^{\prime} .
$$

As was already mentioned, the phase rotation $P_{\alpha}$ leaves $\eta$ invariant, but the symmetries of time translation $T_{z}$ and space translation $S_{y}$ as given in (2.17) are nontrivial. In addition, (3.7) is autonomous, a symmetry which was not present in the original system (2.12), due to the factor $e^{\tau}$ in the right-hand side. Therefore, if $\eta(\tau, \xi)$ is a solution of (3.7), then for every $\tau_{0}, y, z \in \mathbb{R}$ the function

$$
\widehat{\eta}_{\tau_{0}, y, z}(\tau, \xi)=\eta\left(\tau+\log \left(1+\mathrm{e}^{-\tau} z\right)+\tau_{0},\left(\xi+\mathrm{e}^{-\tau / 2} y\right) / \sqrt{1+\mathrm{e}^{-\tau} z}\right)
$$


is also a solution.

If $\eta_{+} \neq \eta_{-}$we may choose for $\eta$ the steady state $\tilde{\eta}$ and obtain a two-dimensional invariant manifold

$$
\mathcal{M}=\{\widetilde{\eta}((\cdot+y) / \sqrt{1+z}) \mid y \in \mathbb{R}, z>-1\}
$$

which is invariant under the flow of (3.7). Using $y$ and $z$ as coordinates on this manifold the reduced flow is $\dot{y}=-\frac{1}{2} y$ and $\dot{z}=-z$. As a consequence, if $\mathbf{K}: \delta \mapsto[a(\widetilde{\eta}(\xi)) \delta]^{\prime \prime}+\frac{\xi}{2} \delta^{\prime}$ is the linearization of (3.7) at the steady state $\widetilde{\eta}$, we have

$$
\mathbf{K} \widetilde{\eta}^{\prime}=-\frac{1}{2} \widetilde{\eta}^{\prime}, \quad \text { and } \quad \mathbf{K}\left(\xi \widetilde{\eta}^{\prime}\right)=-\left(\xi \widetilde{\eta}^{\prime}\right)
$$

since $\left.\partial_{y}\left[S_{y} \widetilde{\eta}\right]\right|_{y=0}=\mathrm{e}^{-\tau / 2} \widetilde{\eta}^{\prime}$ and $\left.\partial_{z}\left[T_{z} \widetilde{\eta}\right]\right|_{z=0}=\mathrm{e}^{-\tau}\left(-\frac{1}{2} \xi \widetilde{\eta}^{\prime}\right)$.

Thus, $\lambda_{0}=-1 / 2$ and $\lambda_{1}=-1$ are eigenvalues of $\mathbf{K}$ for all $\eta_{+}, \eta_{-} \in\left(-\frac{1}{\sqrt{3}}, \frac{1}{\sqrt{3}}\right)$. Since the operators $\mathbf{K}$ and $\mathbf{L}$ are related by $[\mathbf{L} \psi]^{\prime}=\mathbf{K} \psi^{\prime}$, it follows that $\mathbf{L} \widetilde{\eta}=-\frac{1}{2} \widetilde{\eta}$ and $\mathbf{L} \psi_{0}=-\psi_{0}$, where $\psi_{0}(\xi)=2 a(\widetilde{\eta}(\xi)) \widetilde{\eta}^{\prime}(\xi)$ is the primitive of $-\xi \widetilde{\eta}^{\prime}$. This explains the eigenvalue $\lambda_{1}=-1$ of $\mathbf{L}$ in the general case $\eta_{+} \neq \eta_{-}$(Note, however, that $-1 / 2$ is not

an eigenvalue of $\mathbf{L}$, since $\widetilde{\eta}$ does not decay at infinity.) In addition, using Proposition 3.2 and the relation $[\mathbf{L} \psi]^{\prime}=\mathbf{K} \psi^{\prime}$, it is easy to show that the spectrum of $\mathbf{K}$ in the weighted space $X^{\ell}$ is given by $\sigma(\mathbf{K})=\left\{\lambda_{n} \mid n \geq 0\right\} \cup\{z \in \mathbb{C} \mid \operatorname{Re}(z) \leq-1 / 4-\ell / 2\}$.

In fact, the global manifold $\mathcal{M}$ above is a special case of the local spectral manifolds as constructed in Way97. This recent theory for partial differential equations on unbounded domains uses rescaling and weighted norms to generate discrete spectra which then allows for a separation of the spectrum into a finite dimensional part and the remainder. Combining the arguments there and our Proposition 3.2 it should be possible to show that for all $\ell \geq 0$ and all initial data $\eta_{0}$ such that $\operatorname{dist}_{X^{\ell}}\left(\eta_{0}, \mathcal{M}\right)$ is sufficiently small, there exist $y, z \in \mathbb{R}$ such that the solution $\eta$ of (3.7) with $\eta(0, \cdot)=\eta_{0}$ satisfies

$$
\left\|\eta(\tau, \cdot)-\widetilde{\eta}\left(\left(\cdot+\mathrm{e}^{-\tau / 2} y\right) / \sqrt{1+\mathrm{e}^{-\tau} z}\right)\right\|_{X^{\ell}}=\mathcal{O}\left(\mathrm{e}^{-\alpha \tau)}\right.
$$

as $\tau \rightarrow+\infty$, for all $\alpha<\min \left\{-\lambda_{2}, 1 / 4+\ell / 2\right\}$. Clearly, only the case $\ell>3 / 4$ gives interesting results, since $\alpha>1$ is necessary to isolate the coordinate $z$ properly.

\section{Convergence results}

This section contains the convergence results for the diffusive mixing solutions. We first state our main result for the $(\psi, s)$ system (2.12), and then we transform it back to the original equation (1.1).

\section{Theorem 4.1}

Let $\eta_{+}, \eta_{-} \in\left(-\frac{1}{\sqrt{3}}, \frac{1}{\sqrt{3}}\right), \eta_{+} \neq \eta_{-}$, and let $\widetilde{\eta}$ be given by Theorem 3.1. There exist $\varepsilon_{0}>0$ and $\tau_{0}>0$ such that, for all $\left(\psi_{0}, s_{0}\right) \in \mathrm{H}^{2}(\mathbb{R}) \times \mathrm{H}^{1}(\mathbb{R})$ satisfying $\left\|\psi_{0}\right\|_{\mathrm{H}^{2}}+\left\|s_{0}\right\|_{\mathrm{H}^{1}} \leq \varepsilon_{0}$, the system (2.12) has a unique global solution $(\psi, s) \in \mathcal{C}\left(\left[\tau_{0}, \infty\right), \mathrm{H}^{2}(\mathbb{R}) \times \mathrm{H}^{1}(\mathbb{R})\right)$ satisfying $\left(\psi\left(\tau_{0}\right), s\left(\tau_{0}\right)\right)=\left(\psi_{0}, s_{0}\right)$. Moreover, for all $\gamma \in(0,3 / 4)$ we have

$$
\|\psi(\tau, \cdot)\|_{\mathrm{H}^{2}}=\mathcal{O}\left(\mathrm{e}^{-\gamma \tau}\right) \quad \text { and } \quad\|s(\tau, \cdot)\|_{\mathrm{H}^{1}}=\mathcal{O}\left(\mathrm{e}^{-\tau}\right) \quad \text { as } \tau \rightarrow+\infty .
$$


We shall prove Theorem 4.1 in several steps. In Section 5 we prove a local existence result for the solution $(\psi, s)$ of the system (2.12) in the space $\mathrm{H}^{2}(\mathbb{R}) \times \mathrm{H}^{1}(\mathbb{R})$. In Section 6 we introduce several energy functionals to control the behavior of the solution on the interval of existence. We derive differential inequalities for these functionals which imply, in particular, that for large $\tau_{0}$ every solution arising from sufficiently small data at time $\tau=\tau_{0}$ exists and remains bounded for all subsequent times. Then, using the differential inequalities again, we show that the solution converges exponentially to zero as $\tau \rightarrow+\infty$.

These estimates are rather intricate for two reasons. Firstly, our system is nonautonomous through the factor $\mathrm{e}^{\tau}$ in the equation for $s$. This factor, which amounts to an exponentially growing damping, is very essential since it is responsible for the slaving of the amplitude $\rho$ to the phase derivative $\eta=\widetilde{\eta}+\psi^{\prime}$. Note that the right-hand side of the equation for $s$ does not vanish for $(\psi, s)=0$, hence the origin is only an asymptotic fixed point of (2.12). Secondly, as was already mentioned in Section 2, there is an imbalance in the number of spatial derivatives in (2.12) which forces us to study this system in the rather unusual space $\mathrm{H}^{2}(\mathbb{R}) \times \mathrm{H}^{1}(\mathbb{R})$. In this space, (2.12) behave like a quasilinear system, although the original equation (1.1) is semilinear.

We now translate Theorem 4.1 back to the original variable $u=\sqrt{1-\left(\widetilde{\eta}+\psi^{\prime}\right)^{2}} \mathrm{e}^{\sqrt{t}[\tilde{N}+\psi]}$. Since the function $u$ does not decay as $|x| \rightarrow \infty$, it is convenient to use the uniformly local Sobolev spaces $\mathrm{H}_{\mathrm{lu}}^{k}(\mathbb{R})$, see MiS95, Mi97a]. These function spaces are equipped with two different norms: a weighted norm $\left(\mathrm{H}_{w}^{k}\right.$ norm $)$ and a uniformly local norm $\left(\mathrm{H}_{\mathrm{lu}}^{k}\right.$ norm). Without loss of generality, we choose here the weight function $w(x)=1 /\left(1+x^{2}\right)$. For $k \in \mathbb{N}$, we define

$$
\|u\|_{\mathrm{H}_{w}^{k}}^{2}=\int_{\mathbb{R}} w(x)\left(|u(x)|^{2}+\ldots+\left|\partial_{x}^{k} u(x)\right|^{2}\right) \mathrm{d} x, \quad\|u\|_{\mathrm{H}_{\mathrm{lu}}^{k}}=\sup _{y \in \mathbb{R}}\|u(\cdot+y)\|_{\mathrm{H}_{w}^{k}} .
$$

In particular, one has $\mathcal{C}_{\mathrm{b}}^{1}(\mathbb{R}) \subset \mathrm{H}_{\text {lu }}^{1}(\mathbb{R}) \subset \mathrm{L}^{\infty}(\mathbb{R})$ and $\|u\|_{\mathrm{L}^{\infty}} \leq C\|u\|_{\mathrm{H}_{\mathrm{lu}}^{1}(\mathbb{R})}$ for some $C>0$.

For all $u_{0} \in \mathrm{H}_{\text {lu }}^{k}(\mathbb{R}), k \in \mathbb{N}$, the Ginzburg-Landau equation (1.1), has a unique global solution $u \in \mathcal{C}\left([0, \infty), \mathrm{H}_{\mathrm{lu}}^{k}(\mathbb{R})\right)$ with $u(0, \cdot)=u_{0}$ (see Col94, MiS95). We now consider special initial conditions which are close to the mixing profiles (1.6) associated with $\widetilde{\eta}$. Our result is

\section{Theorem 4.2}

Let $\eta_{+}, \eta_{-} \in\left(-\frac{1}{\sqrt{3}}, \frac{1}{\sqrt{3}}\right), \eta_{+} \neq \eta_{-}$, and let $\widetilde{U}$ be given by (1.6). There exist $t_{0}>0$ and $\varepsilon>0$ such that, for all $u_{0} \in \mathrm{H}_{\mathrm{lu}}^{2}(\mathbb{R})$ satisfying $\left\|u_{0}-\widetilde{U}\left(t_{0}, \cdot\right)\right\|_{\mathrm{H}^{2}} \leq \varepsilon$, the unique solution $u$ of (1.1) in $\mathrm{H}_{\mathrm{lu}}^{2}(\mathbb{R})$ with $u(0, \cdot)=u_{0}$ satisfies, for all $\nu \in(0,1)$,

$$
\left\|u(t, \cdot)-\widetilde{U}\left(t_{0}+t, \cdot\right)\right\|_{\mathrm{H}_{1 \mathrm{u}}^{1}}=\mathcal{O}\left(t^{-\nu / 4}\right), \quad\left\||u(t, \cdot)|-\left|\widetilde{U}\left(t_{0}+t, \cdot\right)\right|\right\|_{\mathrm{H}_{\mathrm{lu}}^{1}}=\mathcal{O}\left(t^{-3 \nu / 4}\right),
$$

as $t \rightarrow+\infty$.

\section{Remarks.}

1) The loss of regularity from $\mathrm{H}^{2}$ to $\mathrm{H}^{1}$ is generated by the imbalance in the number of derivatives of $\psi$ and $s$ in (2.12): we need an $\mathrm{H}^{2}$ condition on the data to ensure that $\psi \in \mathrm{H}^{2}$, but since $s \in \mathrm{H}^{1}$ we can only recover an $\mathrm{H}^{1}$ regularity in our results.

2) The initial perturbations are assumed to be small in the $\mathrm{H}^{2}$ norm, but our convergence results are formulated in the uniformly local norm $\|\cdot\|_{\mathrm{H}_{\mathrm{lu}}^{1}}$ only, because we want to keep 
the optimal decay rate in time. Indeed, since the estimates for $(\psi, s)$ are obtained in $\mathrm{H}^{k}$ norms with respect to the diffusive variable $\xi=x / \sqrt{t}$ (Theorem 4.1), we lose a factor $t^{1 / 4}$ when transforming the results back to the corresponding $\mathrm{H}^{k}$ norms with respect to the original variable $x$. For instance, we find $\left\||u(t, \cdot)|-\left|\widetilde{U}\left(t_{0}+t, \cdot\right)\right|\right\|_{\mathrm{H}^{1}}=\mathcal{O}\left(t^{-\nu / 2}\right)$, since the amplitudes are just rescaled but do not involve a prefactor $\sqrt{t}$ like for the phase $\varphi$. However, it is not possible to give a definite decay rate for $\left\|u(t, \cdot)-\widetilde{U}\left(t_{0}+t, \cdot\right)\right\|_{\mathrm{H}^{1}}$ unless we impose further spatial decay properties. Assuming the perturbation to lie in $X^{\ell}$ (together with the first two derivatives) it is possible to improve the decay in (4.1) to $\mathcal{O}\left(\mathrm{e}^{-\alpha \tau}\right)$ for all $\alpha<\min \{1,3 / 4+\ell / 2\}$ (see [BrK92] and the arguments at the end of Section 3 above). Then $\left\|u(t, \cdot)-\widetilde{U}\left(t_{0}+t, \cdot\right)\right\|_{\mathrm{H}^{1}}=\mathcal{O}\left(t^{3 / 4}-\alpha\right)$ can be concluded, which gives a positive decay rate whenever $\ell>1 / 2$.

Proof: Let $t_{0}=e^{\tau_{0}}$, where $\tau_{0}$ is given by Theorem 4.1. If $u_{0} \in \mathrm{H}_{\mathrm{lu}}^{2}(\mathbb{R})$ satisfies $\| u_{0}-$ $\widetilde{U}\left(t_{0}, \cdot\right) \|_{\mathrm{H}_{\mathrm{lu}}^{2}} \leq \varepsilon$ for some sufficiently small $\varepsilon>0$, there exists a unique pair of functions $\left(\psi_{0}, s_{0}\right) \in \mathrm{H}^{2}(\mathbb{R}) \times \mathrm{H}^{1}(\mathbb{R})$ such that

$$
u_{0}\left(\sqrt{t_{0}} \xi\right)=\sqrt{1-\left(\widetilde{\eta}(\xi)+\psi_{0}^{\prime}(\xi)\right)^{2}} \mathrm{e}^{s_{0}(\xi) / 2} \mathrm{e}^{\mathrm{i} \sqrt{t_{0}}\left[\tilde{N}(\xi)+\psi_{0}(\xi)\right]}, \quad \xi \in \mathbb{R} .
$$

In addition, one has $\left\|\psi_{0}\right\|_{\mathrm{H}^{2}}+\left\|s_{0}\right\|_{\mathrm{H}^{1}} \leq C \varepsilon$ for some $C>0$. Thus, if $C \varepsilon \leq \varepsilon_{0}$, Theorem 4.1 shows that the unique solution of (2.12) in $\mathrm{H}^{2}(\mathbb{R}) \times \mathrm{H}^{1}(\mathbb{R})$ with initial data $\left(\psi_{0}, s_{0}\right)$ satisfies $\|\psi(\tau, \cdot)\|_{\mathrm{H}^{2}}+\|s(\tau, \cdot)\|_{\mathrm{H}^{1}}=\mathcal{O}\left(\mathrm{e}^{-\gamma \tau}\right)$ as $\tau \rightarrow+\infty$, for all $\gamma<3 / 4$. In particular, we have for all $\nu<1$ :

$$
\begin{aligned}
& \int_{\mathbb{R}} w(x)\left(\psi^{2}+\psi^{\prime 2}+s^{2}\right)(x / \sqrt{t}, \log t) \mathrm{d} x \leq C \sup _{\xi \in \mathbb{R}}\left(\psi^{2}+\psi^{\prime 2}+s^{2}\right)(\xi, \log t)=\mathcal{O}\left(t^{-3 \nu / 2}\right), \\
& \int_{\mathbb{R}} w(x)\left(\psi^{\prime \prime 2}+s^{\prime 2}\right)(x / \sqrt{t}, \log t) \mathrm{d} x \leq C \sqrt{t} \int_{\mathbb{R}}\left(\psi^{\prime \prime 2}+s^{\prime 2}\right)(\xi, \log t) \mathrm{d} \xi=\mathcal{O}\left(t^{-\nu}\right),
\end{aligned}
$$

where $w$ is the weight function $1 /\left(1+x^{2}\right)$ or any translate of it. Using these results, it is straightforward to verify that the unique solution $u(t, x)$ of $(1.1)$ in $\mathrm{H}_{\mathrm{lu}}^{2}(\mathbb{R})$ with initial data $u_{0}$ is given by

$u(t, x)=\sqrt{1-\left(\widetilde{\eta}(\xi)+\psi^{\prime}(\tau, \xi)\right)^{2}} \mathrm{e}^{s(\tau, \xi) / 2} \mathrm{e}^{\mathrm{i} \sqrt{t}[\tilde{N}(\xi)+\psi(\tau, \xi)]}, \quad$ with $\tau=\log \left(t+t_{0}\right), \quad \xi=\frac{x}{\sqrt{t+t_{0}}}$,

and that the decay estimates (4.3) are satisfied.

Theorem 4.2 implies that, up to a time-dependent phase, the solution $u(t, x)$ converges uniformly on compact sets to the stationary solution $\sqrt{1-\eta_{*}^{2}} \mathrm{e}^{\mathrm{i} \eta_{*} x}$ as $t \rightarrow+\infty$, where

$\eta_{*}=\widetilde{\eta}(0)$. Indeed, setting $\varphi_{*}=\widetilde{N}(0)=\int_{-\infty}^{0}\left(\widetilde{\eta}(\xi)-\eta_{-}\right) \mathrm{d} \xi \neq 0$ as in Theorem 3.1, we have the following result (see also [BrK92]):

\section{Corollary 4.3}

Under the assumptions of Theorem 4.2, we have for all $x_{0}>0$ and all $\nu \in(0,1)$ the estimate

$$
\sup _{|x| \leq x_{0}}\left|u(t, x)-\sqrt{1-\eta_{*}^{2}} \mathrm{e}^{\mathrm{i}\left[\sqrt{t} \varphi_{*}+\eta_{*} x\right]}\right|=\mathcal{O}\left(t^{-\nu / 4}\right), \quad t \rightarrow+\infty
$$

where $\eta_{*}=\widetilde{\eta}(0)$ and $\varphi_{*}=\widetilde{N}(0)$. 
Proof: Since $\widetilde{\eta}(x / \sqrt{t})=\eta_{*}+\mathcal{O}\left(t^{-1 / 2}\right)$ and $\sqrt{t} \widetilde{N}(x / \sqrt{t})=\sqrt{t} \varphi_{*}+\eta_{*} x+\mathcal{O}\left(t^{-1 / 2}\right)$ uniformly for $|x| \leq x_{0}$, this result follows immediately from the first estimate in (4.3).

Remark. In the case where both $\eta_{+}, \eta_{-}$are close to zero, the estimate (4.4) is obtained in BrK92 with the better decay rate $\mathcal{O}\left(t^{-\nu / 2}\right)$ for the remainder. As was already mentioned in the previous remark, this is because we allow for initial perturbations with only $\mathrm{L}^{2}$ decay at infinity, while in [BrK92] stronger norms are used which imply in particular that the perturbations lie in $X^{\ell}$ with $\ell \geq 1$.

\section{Local existence}

In this section, we prove a local existence result for the solutions $(\psi, s)$ of the system (2.12) in the space $\mathrm{H}^{2}(\mathbb{R}) \times \mathrm{H}^{1}(\mathbb{R})$. Although it originates from the simple Ginzburg-Landau equation (1.1), this system in nonautonomous due to the change of variables (2.8). In addition, the nonlinear transformation (2.11) forces us to study it in the unbalanced space $\mathrm{H}^{2}(\mathbb{R}) \times \mathrm{H}^{1}(\mathbb{R})$, where it behaves like a quasilinear system. For these reasons, we have to take special care of the regularity of the solutions and the length of the local existence intervals.

Throughout this section, we assume that $\eta_{+}, \eta_{-} \in\left(-\frac{1}{\sqrt{3}}, \frac{1}{\sqrt{3}}\right)$ and that $\widetilde{\eta}$ is given by Theorem 3.1. Our result is:

\section{Proposition 5.1}

There exist $\varepsilon_{1}>0, T_{1}>0$, and $K_{1} \geq 1$ such that, for all $\tau_{1} \geq 0$ and all $\left(\psi_{1}, s_{1}\right) \in$ $\mathrm{H}^{2}(\mathbb{R}) \times \mathrm{H}^{1}(\mathbb{R})$ such that $\left\|\psi_{1}\right\|_{\mathrm{H}^{2}}+\left\|s_{1}\right\|_{\mathrm{H}^{1}} \leq \varepsilon_{1}$, the system $(2.12)$ has a unique solution $(\psi, s) \in \mathcal{C}\left(\left[\tau_{1}, \tau_{2}\right], \mathrm{H}^{2}(\mathbb{R}) \times \mathrm{H}^{1}(\mathbb{R})\right)$ satisfying $\left(\psi\left(\tau_{1}\right), s\left(\tau_{1}\right)\right)=\left(\psi_{1}, s_{1}\right)$, where $\tau_{2}=$ $\tau_{1}+\log \left(1+T_{1} \mathrm{e}^{-\tau_{1}}\right)$. This solution depends continuously on the initial data $\left(\psi_{1}, s_{1}\right)$ in $\mathrm{H}^{2}(\mathbb{R}) \times \mathrm{H}^{1}(\mathbb{R})$, uniformly in $\tau \in\left[\tau_{1}, \tau_{2}\right]$. Moreover, the bound

$$
\|\psi(\tau)\|_{\mathrm{H}^{2}}+\|s(\tau)\|_{\mathrm{H}^{1}} \leq K_{1}\left(\left\|\psi_{1}\right\|_{\mathrm{H}^{2}}+\left\|s_{1}\right\|_{\mathrm{H}^{1}}+\mathrm{e}^{\tau_{1}}\left(\tau-\tau_{1}\right)\right)
$$

holds for all $\tau \in\left[\tau_{1}, \tau_{2}\right]$.

\section{Remarks.}

1) Here and in the sequel, we simply write $\psi(\tau), s(\tau)$ instead of $\psi(\tau, \cdot), s(\tau, \cdot)$ when no confusion is possible.

2) In particular, Proposition 5.1 implies that, if $(\psi, s) \in \mathcal{C}\left(\left[\tau_{1}, \tau^{*}\right), \mathrm{H}^{2}(\mathbb{R}) \times \mathrm{H}^{1}(\mathbb{R})\right)$ is a maximal solution of (2.12) which satisfies $\|\psi(\tau)\|_{\mathrm{H}^{2}}+\|s(\tau)\|_{\mathrm{H}^{1}} \leq \varepsilon_{1}$ for all $\tau \in\left[\tau_{1}, \tau^{*}\right)$, then actually $\tau^{*}=+\infty$, i.e. the solution can be continued to the whole interval $\left[\tau_{1},+\infty\right)$. 3) The proof shows that the solution $\psi(\tau, \xi), s(\tau, \xi)$ of (2.12) is a $\mathcal{C}^{\infty}$ function of $\tau, \xi$ for all $\tau>\tau_{1}$. However, it is not true in general that $(\psi, s) \in \mathcal{C}^{k}\left(\left(\tau_{1}, \tau_{2}\right], \mathrm{H}^{2}(\mathbb{R}) \times \mathrm{H}^{1}(\mathbb{R})\right)$ for $k>0$, unless we assume in addition that $\psi_{1}(\xi), s_{1}(\xi)$ decay sufficiently fast as $|\xi| \rightarrow \infty$.

Proof: Instead of working directly on (2.12), we shall use the change of variables (2.9), (2.11) and solve the corresponding initial value problem for the simpler system (2.2). Let $\tau_{1} \geq 0$, and let $\left(\psi_{1}, s_{1}\right) \in \mathrm{H}^{2}(\mathbb{R}) \times \mathrm{H}^{1}(\mathbb{R})$ be initial data for (2.12) at time $\tau=\tau_{1}$ satisfying 
$\left\|\psi_{1}\right\|_{\mathrm{H}^{2}}+\left\|s_{1}\right\|_{\mathrm{H}^{1}}=\varepsilon$ for some $\varepsilon \leq 1 / 4$. The corresponding initial data for (2.2) are given by

$$
\varphi\left(t_{1}, x\right)=\varphi_{L}(x)+\bar{\varphi}_{1}(x), \quad r\left(t_{1}, x\right)=r_{L}(x)+\bar{r}_{1}(x),
$$

where $t_{1}=\mathrm{e}^{\tau_{1}}, L=\mathrm{e}^{\tau_{1} / 2}, \varphi_{L}(x)=L \tilde{N}(x / L), r_{L}(x)=\left(1-\widetilde{\eta}(x / L)^{2}\right)^{1 / 2}$, and

$$
\bar{\varphi}_{1}(x)=L \psi_{1}(x / L), \quad \bar{r}_{1}(x)=\left(1-\left(\widetilde{\eta}(x / L)+\psi_{1}^{\prime}(x / L)\right)^{2}\right)^{1 / 2} \mathrm{e}^{\frac{1}{2} s_{1}(x / L)}-r_{L}(x) .
$$

A direct calculation shows that there exists a constant $C_{1}>0$ (independent of $L$ ) such that $\left\|\bar{\varphi}_{1}\right\|_{\mathrm{H}_{L}^{2}}+\left\|\bar{r}_{1}\right\|_{\mathrm{H}_{L}^{1}} \leq C_{1}\left(\left\|\psi_{1}\right\|_{\mathrm{H}^{2}}+\left\|s_{1}\right\|_{\mathrm{H}^{1}}\right)$, where $\mathrm{H}_{L}^{2}, \mathrm{H}_{L}^{1}$ are the Sobolev spaces $\mathrm{H}^{2}(\mathbb{R})$, $\mathrm{H}^{1}(\mathbb{R})$ equipped with the $L$-dependent norms

$$
\|\bar{\varphi}\|_{\mathrm{H}_{L}^{2}}^{2}=L^{-3}\|\bar{\varphi}\|_{\mathrm{L}^{2}}^{2}+L^{-1}\left\|\bar{\varphi}^{\prime}\right\|_{\mathrm{L}^{2}}^{2}+L\left\|\bar{\varphi}^{\prime \prime}\right\|_{\mathrm{L}^{2}}^{2}, \quad\|\bar{r}\|_{\mathrm{H}_{L}^{1}}^{2}=L^{-1}\|\bar{r}\|_{\mathrm{L}^{2}}^{2}+L\left\|\bar{r}^{\prime}\right\|_{\mathrm{L}^{2}}^{2} .
$$

Motivated by (5.2), we look for a solution of (2.2) of the form $\varphi(t, x)=\varphi_{L}(x)+\bar{\varphi}(t, x)$, $r(t, x)=r_{L}(x)+\bar{r}(t, x)$, where $\bar{\varphi}, \bar{r}$ satisfy the evolution system

$$
\begin{aligned}
\partial_{t} \bar{\varphi} & =\partial_{x}^{2} \bar{\varphi}+\varphi_{L}^{\prime \prime}+2\left(r_{L}+\bar{r}\right)^{-1}\left(r_{L}^{\prime}+\partial_{x} \bar{r}\right)\left(\varphi_{L}^{\prime}+\partial_{x} \bar{\varphi}\right), \\
\partial_{t} \bar{r} & =\partial_{x}^{2} \bar{r}+r_{L}^{\prime \prime}-\left(\bar{r}\left(r_{L}+2 \bar{r}\right)+\left(\partial_{x} \bar{\varphi}\right)\left(2 \varphi_{L}^{\prime}+\partial_{x} \bar{\varphi}\right)\right)\left(r_{L}+\bar{r}\right)
\end{aligned}
$$

together with the initial condition $\left(\bar{\varphi}\left(t_{1}\right), \bar{r}\left(t_{1}\right)\right)=\left(\bar{\varphi}_{1}, \bar{r}_{1}\right) \in \mathrm{H}_{L}^{2} \times \mathrm{H}_{L}^{1}$. If $C_{1} \varepsilon \leq 1 / 4$, Lemma 5.2 below shows that this initial value problem has a unique solution $(\bar{\varphi}, \bar{r}) \in$ $\mathcal{C}\left(\left[t_{1}, t_{1}+T\right], \mathrm{H}_{L}^{2} \times \mathrm{H}_{L}^{1}\right)$, for some $T>0$ (independent of $\left.L\right)$. This solution depends continuously on the initial data $\left(\bar{\varphi}\left(t_{1}\right), \bar{r}\left(t_{1}\right)\right)$ in $\mathrm{H}_{L}^{2} \times \mathrm{H}_{L}^{1}$, uniformly in $t \in\left[t_{1}, t_{1}+T\right]$. In addition, there exist $C_{2}>0, C_{3} \geq 1$ (independent of $L$ ) such that

$$
\|\bar{\varphi}(t)\|_{\mathrm{H}_{L}^{2}}+\|\bar{r}(t)\|_{\mathrm{H}_{L}^{1}} \leq C_{2}\left(t-t_{1}\right)+C_{3} C_{1} \varepsilon,
$$

for all $t \in\left[t_{1}, t_{1}+T\right]$. Finally, due to the parabolic regularization, $\bar{\varphi}(t, x)$ and $\bar{r}(t, x)$ are $\mathcal{C}^{\infty}$ functions of $t, x$ for all $t>t_{1}$.

Having constructed the solution $(\bar{\varphi}, \bar{r})$ of (5.4), we now return to the variables $(\psi, s)$ defined by the relations (2.8), (2.11). Setting $\sigma=\tau-\tau_{1}$ and using the definitions above of $L, \varphi_{L}, r_{L}$, we arrive at the expressions

$$
\begin{aligned}
\psi\left(\tau_{1}+\sigma, \xi\right)= & \mathrm{e}^{-\sigma / 2} \widetilde{N}\left(\xi \mathrm{e}^{\sigma / 2}\right)-\widetilde{N}(\xi)+\mathrm{e}^{-\sigma / 2} L^{-1} \bar{\varphi}\left(\xi L \mathrm{e}^{\sigma / 2}, L^{2} \mathrm{e}^{\sigma}\right) \\
s\left(\tau_{1}+\sigma, \xi\right)= & 2 \log \left[R(\xi)^{-1} R\left(\xi \mathrm{e}^{\sigma / 2}\right)\right]+2 \log \left[1+R\left(\xi \mathrm{e}^{\sigma / 2}\right)^{-1} \bar{r}\left(\xi L \mathrm{e}^{\sigma / 2}, L^{2} \mathrm{e}^{\sigma}\right)\right] \\
& -\log \left[1-2 R(\xi)^{-2}\left(\widetilde{\eta}^{\prime}(\xi) \psi^{\prime}(\xi, \tau)+\psi^{\prime}(\xi, \tau)^{2}\right)\right]
\end{aligned}
$$

where $0 \leq \sigma \leq \sigma_{1}=\log \left(1+T \mathrm{e}^{-\tau_{1}}\right)$ and $R(\xi)=\sqrt{1-\widetilde{\eta}(\xi)^{2}}$. If we assume that $C_{2} T+$ $C_{1} C_{3} \varepsilon \leq 1 / 4$, then a direct calculation shows that $(\psi, s) \in \mathcal{C}\left(\left[\tau_{1}, \tau_{1}+\sigma_{1}\right], \mathrm{H}^{2} \times \mathrm{H}^{1}\right)$ satisfies, for all $\tau \in\left[\tau_{1}, \tau_{1}+\sigma_{1}\right]$,

$$
\|\psi(\tau)\|_{\mathrm{H}^{2}}+\|s(\tau)\|_{\mathrm{H}^{1}} \leq C_{4}\left(\tau-\tau_{1}\right)+C_{5}\left(\left\|\bar{\varphi}\left(\mathrm{e}^{\tau}\right)\right\|_{\mathrm{H}_{L}^{2}}+\left\|\bar{r}\left(\mathrm{e}^{\tau}\right)\right\|_{\mathrm{H}_{L}^{1}}\right),
$$

where $C_{4}>0$ and $C_{5} \geq 1$ are independent of $L$ (or, equivalently, of $\tau_{1}$ ). The proof of (5.7) relies on the properties of $\widetilde{\eta}(\xi)$ listed in Theorem 3.1 and on the fact that the dilation $\lambda \mapsto \varphi(\lambda \cdot)$ is a continuous operation in $\mathrm{H}^{k}(\mathbb{R}), k \in \mathbb{N}$. The uniformity in $L$ of the constants $C_{4}, C_{5}$ follows from the definition of the scaled norms (5.3) and from the fact that $0 \leq \sigma \leq T$ uniformly in $L$. 
By construction, $(\psi, s) \in \mathcal{C}\left(\left[\tau_{1}, \tau_{1}+\sigma_{1}\right], \mathrm{H}^{2} \times \mathrm{H}^{1}\right)$ is a solution of the system (2.12) satisfying $\left(\psi\left(\tau_{1}\right), s\left(\tau_{1}\right)\right)=\left(\psi_{1}, s_{1}\right)$. The uniqueness of this solution and its continuous dependence on the data follow directly from the corresponding properties of the system (5.4), see Lemma 5.2. Finally, combining (5.5), (5.7), we obtain

$$
\|\psi(\tau)\|_{\mathrm{H}^{2}}+\|s(\tau)\|_{\mathrm{H}^{1}} \leq C_{1} C_{3} C_{5} \varepsilon+\left(C_{4}+C_{2} C_{5} \mathrm{e}^{\tau}\right)\left(\tau-\tau_{1}\right),
$$

for all $\tau \in\left[\tau_{1}, \tau_{1}+\sigma_{1}\right]$. Therefore, we see that (5.1) holds if we set, for example,

$$
T_{1}=\min \left(T, \frac{1}{8 C_{2}}\right), \quad \varepsilon_{1}=\frac{1}{8 C_{1} C_{3}}, \quad K_{1}=\max \left(C_{1} C_{3} C_{5}, C_{4}+C_{2} C_{5}\left(1+T_{1}\right)\right) .
$$

This concludes the proof of Proposition 5.1.

Proposition 5.1 relies on a local existence result for the solutions of (5.4) which is the content of the next Lemma. Since the system (5.4) is autonomous, we assume here (without loss of generality) that the initial time is $t_{1}=0$. We recall that $\mathrm{H}_{L}^{2}, \mathrm{H}_{L}^{1}$ are the function spaces defined by the norms (5.3).

\section{Lemma 5.2}

There exist $T>0, C_{2}>0, C_{3} \geq 1$ such that, for all $L \geq 1$ and for all $\left(\bar{\varphi}_{1}, \bar{r}_{1}\right) \in$ $\mathrm{H}_{L}^{2} \times \mathrm{H}_{L}^{1}$ such that $\left\|\bar{\varphi}_{1}\right\|_{\mathrm{H}_{L}^{2}}+\left\|\bar{r}_{1}\right\|_{\mathrm{H}_{L}^{1}} \leq 1 / 4$, the system (5.4) has a unique solution $(\bar{\varphi}, \bar{r}) \in$ $\mathcal{C}\left([0, T], \mathrm{H}_{L}^{2} \times \mathrm{H}_{L}^{1}\right)$ satisfying $(\bar{\varphi}(0), \bar{r}(0))=\left(\bar{\varphi}_{1}, \bar{r}_{1}\right)$. This solution depends continuously on the initial data $\left(\bar{\varphi}_{1}, \bar{r}_{1}\right)$ in $\mathrm{H}_{L}^{2} \times \mathrm{H}_{L}^{1}$, uniformly in $t \in[0, T]$. Moreover, the bound

$$
\|\bar{\varphi}(t)\|_{\mathrm{H}_{L}^{2}}+\|\bar{r}(t)\|_{\mathrm{H}_{L}^{1}} \leq C_{2} t+C_{3}\left(\left\|\bar{\varphi}_{1}\right\|_{\mathrm{H}_{L}^{2}}+\left\|\bar{r}_{1}\right\|_{\mathrm{H}_{L}^{1}}\right),
$$

holds for all $t \in[0, T]$.

Proof: Lemma 5.2 is a standard local existence result, except that we have to control the dependence on the scaling parameter $L \geq 1$, and that we need one more derivative for $\bar{\varphi}$ than for $\bar{r}$. Because of this imbalance, the apparently semilinear equation (5.4) behaves in fact like a quasilinear system. In particular, the constant $C_{3}$ in (5.8) cannot be replaced by 1 .

Throughout the proof, we write $(\varphi, r)$ instead of $(\bar{\varphi}, \bar{r})$ for simplicity. The system (5.4) becomes

$$
\partial_{t} \varphi=\partial_{x}^{2} \varphi+F_{L}(\varphi, r), \quad \partial_{t} r=\partial_{x}^{2} r+G_{L}(\varphi, r),
$$

where

$$
\begin{aligned}
& F_{L}(\varphi, r)=\varphi_{L}^{\prime \prime}+2\left(r_{L}+r\right)^{-1}\left(r_{L}^{\prime}+\partial_{x} r\right)\left(\varphi_{L}^{\prime}+\partial_{x} \varphi\right), \\
& G_{L}(\varphi, r)=r_{L}^{\prime \prime}-\left(r\left(r_{L}+2 r\right)+\left(\partial_{x} \varphi\right)\left(2 \varphi_{L}^{\prime}+\partial_{x} \varphi\right)\right)\left(r_{L}+r\right) .
\end{aligned}
$$

To avoid the difficulty related to the imbalance of derivatives, we first prove the existence of a unique local solution of (5.9) in a subspace of $\mathrm{H}_{L}^{2} \times \mathrm{H}_{L}^{1}$ in which the derivatives are balanced. This will be done using a standard contraction mapping argument.

Let $L \geq 1$, and let $\left(\bar{\varphi}_{1}, \bar{r}_{1}\right) \in \mathrm{H}_{L}^{2} \times \mathrm{H}_{L}^{1}$ satisfying $m_{1} \equiv\left\|\bar{\varphi}_{1}\right\|_{\mathrm{H}_{L}^{2}}+\left\|\bar{r}_{1}\right\|_{\mathrm{H}_{L}^{1}} \leq 1 / 4$. For any $T>0$, we denote by $X(T)$ the Banach space

$$
X(T)=\left\{(\varphi, r) \mid \varphi, r \in \mathcal{C}\left([0, T], \mathrm{H}_{L}^{1}\right), \partial_{x} \varphi \in \mathcal{C}\left([0, T], \mathrm{L}^{\infty}\right), \partial_{x}^{2} \varphi \in \mathrm{L}^{2}\left([0, T], \mathrm{L}^{2}\right)\right\},
$$


equipped with the norm $\|(\varphi, r)\|_{X(T)}$ given by

$$
\max \left\{\sup _{0 \leq t \leq T}\left(\|r(t)\|_{\mathrm{H}_{L}^{1}}+L^{-1}\|\varphi(t)\|_{\mathrm{H}_{L}^{1}}\right), \sup _{0 \leq t \leq T}\left\|\partial_{x} \varphi(t)\right\|_{\mathrm{L}^{\infty}}, \quad\left(L \int_{0}^{T}\left\|\partial_{x}^{2} \varphi(s)\right\|_{\mathrm{L}^{2}}^{2} \mathrm{~d} s\right)^{1 / 2}\right\} .
$$

We note $\tilde{\varphi}(t)=\mathrm{e}^{t \partial_{x}^{2}} \bar{\varphi}_{1}, \tilde{r}(t)=\mathrm{e}^{t \partial_{x}^{2}} \bar{r}_{1}$, where $\mathrm{e}^{t \partial_{x}^{2}}$ is the heat kernel. Then $(\tilde{\varphi}, \tilde{r}) \in X(T)$ and $\|(\tilde{\varphi}, \tilde{r})\|_{X(T)} \leq 1 / 4$ for any $T \leq 1$. Let $\mathcal{B}(T)$ be the ball of radius $1 / 4$ centered at $(\tilde{\varphi}, \tilde{r})$ in $X(T)$. For all $(\varphi, r) \in \mathcal{B}(T)$, we define

$$
\hat{\varphi}(t)=\int_{0}^{t} \mathrm{e}^{(t-s) \partial_{x}^{2}} F_{L}(\varphi(s), r(s)) \mathrm{d} s, \quad \hat{r}(t)=\int_{0}^{t} \mathrm{e}^{(t-s) \partial_{x}^{2}} G_{L}(\varphi(s), r(s)) \mathrm{d} s .
$$

We shall show that $(\hat{\varphi}, \hat{r}) \in X(T)$ and $\|(\hat{\varphi}, \hat{r})\|_{X(T)} \leq 1 / 4$ if $T$ is sufficiently small (uniformly in $L)$. Indeed, since $(\varphi, r) \in \mathcal{B}(T)$, we have $r_{L}(x)+r(x, t) \geq \inf _{x} r_{L}(x)-\|r(t)\|_{\mathrm{L}^{\infty}} \geq$ $\sqrt{2 / 3}-1 / 2 \geq 1 / 4$ for all $x \in \mathbb{R}, t \in[0, T]$. As a consequence, we have

$$
\begin{aligned}
\left\|F_{L}(\varphi, r)\right\|_{\mathrm{L}^{2}} & \leq\left\|\varphi_{L}^{\prime \prime}\right\|_{\mathrm{L}^{2}}+8\left\|r_{L}^{\prime}+\partial_{x} r\right\|_{\mathrm{L}^{2}}\left\|\varphi_{L^{\prime}}^{\prime}+\partial_{x} \varphi\right\|_{\mathrm{L}^{\infty}} \leq C L^{-1 / 2} \\
\left\|G_{L}(\varphi, r)\right\|_{\mathrm{L}^{2}} & \leq\left\|r_{L}^{\prime \prime}\right\|_{\mathrm{L}^{2}}+\left(\|r\|_{\mathrm{L}^{2}}\left\|r_{L}+2 r\right\|_{\mathrm{L}^{\infty}}+\left\|\partial_{x} \varphi\right\|_{\mathrm{L}^{2}}\left\|2 \varphi_{L}^{\prime}+\partial_{x} \varphi\right\|_{\mathrm{L}^{\infty}}\right)\left\|r_{L}+r\right\|_{\mathrm{L}^{\infty}} \\
& \leq C L^{-3 / 2}+C L^{1 / 2} \leq 2 C L^{1 / 2}
\end{aligned}
$$

for some $C>0$ (independent of $L$ ). Therefore, using standard estimates for the heat kernel, we obtain for all $t \in[0, T]$ :

$$
\begin{aligned}
& \|\hat{\varphi}(t)\|_{L^{2}} \leq C L^{-1 / 2} t, \quad\left\|\partial_{x} \hat{\varphi}(t)\right\|_{L^{2}} \leq C L^{-1 / 2} \sqrt{t}, \quad\left\|\partial_{x} \hat{\varphi}(t)\right\|_{L^{\infty}} \leq C L^{-1 / 2} t^{1 / 4}, \\
& \|\hat{r}(t)\|_{L^{2}} \leq C L^{1 / 2} t, \quad \int_{0}^{t}\left\|\partial_{x}^{2} \hat{\varphi}(s)\right\|_{L^{2}}^{2} \mathrm{~d} s \leq C L^{-1} t .
\end{aligned}
$$

Moreover, differentiating $G_{L}$ with respect to $x$ and proceeding as above, we find $\left\|\partial_{x} G_{L}(\varphi, r)\right\|_{\mathrm{L}^{2}} \leq C L^{-1 / 2}+C\left\|\partial_{x}^{2} \varphi\right\|_{\mathrm{L}^{2}}$, hence

$$
\left\|\partial_{x} \hat{r}(t)\right\|_{\mathrm{L}^{2}} \leq\left\{t \int_{0}^{t}\left\|\partial_{x} G_{L}(\varphi, r)\right\|_{\mathrm{L}^{2}}^{2} \mathrm{~d} s\right\}^{1 / 2} \leq C L^{-1 / 2} t+C L^{-1 / 2} \sqrt{t} \leq C L^{-1 / 2} \sqrt{t},
$$

for all $t \in[0, T]$. From (5.12), (5.13), we conclude that $\|(\hat{\varphi}, \hat{r})\|_{X(T)} \leq C T^{1 / 4}$, uniformly in $L \geq 1$. This proves that the mapping $M$ defined by $M(\varphi, r)=(\tilde{\varphi}+\hat{\varphi}, \tilde{r}+\hat{r})$ maps the ball $\mathcal{B}(T)$ into itself, if $T$ is sufficiently small. Using similar estimates, one shows that $M$ is a contraction in $\mathcal{B}(T)$, hence has a unique fixed point $(\varphi, r) \in \mathcal{B}(T)$. By construction, this fixed point is the unique solution of $(5.9)$ in $X(T)$ satisfying $(\varphi(0), r(0))=\left(\bar{\varphi}_{1}, \bar{r}_{1}\right)$, and this solution depends continuously in $X(T)$ on the initial data $\left(\bar{\varphi}_{1}, \bar{r}_{1}\right) \in \mathrm{H}_{L}^{2} \times \mathrm{H}_{L}^{1}$.

It remains to verify that $\partial_{x}^{2} \varphi \in \mathcal{C}\left([0, T], \mathrm{L}^{2}\right)$ and that (5.8) holds. Since $(\varphi, r)=$ $(\tilde{\varphi}, \tilde{r})+(\hat{\varphi}, \hat{r})$, where $(\hat{\varphi}, \hat{r})$ is given by $(5.10)$, and since $\|\tilde{\varphi}(t)\|_{\mathrm{H}_{L}^{2}}+\|\tilde{r}(t)\|_{\mathrm{H}_{L}^{1}} \leq m_{1}=$ $\left\|\bar{\varphi}_{1}\right\|_{\mathrm{H}_{L}^{2}}+\left\|\bar{r}_{1}\right\|_{\mathrm{H}_{L}^{1}}$, it is sufficient to show that $\partial_{x}^{2} \hat{\varphi} \in \mathcal{C}\left([0, T], \mathrm{L}^{2}\right)$ and $\|\hat{\varphi}(t)\|_{\mathrm{H}_{L}^{2}}+\|\hat{r}(t)\|_{\mathrm{H}_{L}^{1}} \leq$ $C\left(m_{1}+t\right)$ for $t \in[0, T]$. Appropriate bounds on $\|\hat{\varphi}\|_{\mathrm{L}^{2}}$ and $\|\hat{r}\|_{\mathrm{L}^{2}}$ are already contained in (5.12). To bound $\left\|\partial_{x} \hat{r}\right\|_{\mathrm{L}^{2}}$, we observe that

$$
\int_{0}^{t}\left\|\partial_{x}^{2} \varphi(s)\right\|_{\mathrm{L}^{2}}^{2} \mathrm{~d} s \leq 2 \int_{0}^{t}\left(\left\|\partial_{x}^{2} \tilde{\varphi}(s)\right\|_{\mathrm{L}^{2}}^{2}+\left\|\partial_{x}^{2} \hat{\varphi}(s)\right\|_{\mathrm{L}^{2}}^{2}\right) \mathrm{d} s \leq C L^{-1} t
$$


by (5.12), hence the estimate $(5.13)$ can be improved as follows:

$$
\left\|\partial_{x} \hat{r}(t)\right\|_{\mathrm{L}^{2}} \leq\left\{t \int_{0}^{t} C\left(L^{-1}+\left\|\partial_{x}^{2} \varphi(s)\right\|_{\mathrm{L}^{2}}^{2}\right) \mathrm{d} s\right\}^{1 / 2} \leq C L^{-1 / 2} t .
$$

To bound $\left\|\partial_{x} \hat{\varphi}\right\|_{\mathrm{L}^{2}}$ and $\left\|\partial_{x}^{2} \hat{\varphi}\right\|_{\mathrm{L}^{2}}$, we need to estimate $\partial_{x} F_{L}$. It is convenient here to write $F_{L}(\varphi, r)=F_{L}(0,0)+\widetilde{F}_{L}(\varphi, r)$, where $F_{L}(0,0)=\varphi_{L}^{\prime \prime}+2 r_{L}^{\prime} \varphi_{L}^{\prime} / r_{L}$. Using (5.12), (5.15), it is straightforward to verify that

$$
\begin{aligned}
& \left\|\partial_{x} F_{L}(0,0)\right\|_{\mathrm{L}^{2}} \leq C L^{-3 / 2}, \quad\left\|\partial_{x}^{2} F_{L}(0,0)\right\|_{\mathrm{L}^{2}} \leq C L^{-5 / 2}, \\
& \left\|\partial_{x} \widetilde{F}_{L}(\varphi, r)\right\|_{\mathrm{L}^{2}} \leq C\left\|\partial_{x}^{2} r\right\|+C\left(L^{-1}+\left\|\partial_{x} r\right\|_{\mathrm{L}^{\infty}}\right)\left(m_{1}+L^{-1 / 2} \sqrt{t}+\left\|\partial_{x}^{2} \hat{\varphi}\right\|_{\mathrm{L}^{2}}\right) .
\end{aligned}
$$

On the other hand, using (5.12), (5.15) as well as various standard estimates for the heat kernel, it is not difficult to show that

$$
\begin{aligned}
& \int_{0}^{t}\left\|\partial_{x}^{2} r(s)\right\|_{L^{2}}^{2} \mathrm{~d} s \leq C L^{-1}\left(m_{1}^{2}+t^{2}\right), \quad \int_{0}^{t}\left\|\partial_{x} r(s)\right\|_{L^{\infty}}^{2} \mathrm{~d} s \leq C L^{-1} \sqrt{t}\left(m_{1}^{2}+t^{2}\right), \\
& \int_{0}^{t}\left\|\partial_{x} r(s)\right\|_{\mathrm{L}^{\infty}}^{2}\left\|\partial_{x}^{2} \hat{\varphi}(s)\right\|_{\mathrm{L}^{2}}^{2} \mathrm{~d} s \leq C L^{-2} \sqrt{t}\left(m_{1}^{2}+t^{2}\right) .
\end{aligned}
$$

Note that the first estimate in (5.17) does not converge to zero as $t \rightarrow 0$, since $r(0)=\bar{r}_{1}$ belongs to $\mathrm{H}_{L}^{1}$ only. Combining (5.16), (5.17), we thus find

$$
\int_{0}^{t}\left\|\partial_{x} \widetilde{F}_{L}(\varphi, r)\right\|_{\mathrm{L}^{2}}^{2} \mathrm{~d} s \leq C L^{-1}\left(m_{1}^{2}+t^{2}\right)+C L^{-2} \int_{0}^{t}\left\|\partial_{x}^{2} \hat{\varphi}\right\|_{\mathrm{L}^{2}}^{2} \mathrm{~d} s .
$$

This result implies that $\partial_{x} F_{L} \in \mathrm{L}^{2}\left([0, T], \mathrm{L}^{2}(\mathbb{R})\right)$ and $\int_{0}^{t}\left\|\partial_{x} F_{L}(\varphi, r)\right\|_{\mathrm{L}^{2}}^{2} \mathrm{~d} s \leq C L^{-1}\left(m_{1}^{2}+\right.$ $\left.t^{2}\right)+C L^{-3} t$ by (5.12), (5.16). It follows that $\hat{\varphi} \in \mathcal{C}\left([0, T], \mathrm{H}_{L}^{2}\right)$ and

$$
\begin{aligned}
& \left\|\partial_{x} \hat{\varphi}(t)\right\|_{\mathrm{L}^{2}} \leq\left\{t \int_{0}^{t}\left\|\partial_{x} F_{L}(\varphi, r)\right\|_{\mathrm{L}^{2}}^{2} \mathrm{~d} s\right\}^{1 / 2} \leq C L^{-1 / 2} \sqrt{t}\left(m_{1}+t\right)+C L^{-3 / 2} t, \\
& \left\|\partial_{x}^{2} \hat{\varphi}(t)\right\|_{\mathrm{L}^{2}} \leq\left\{\int_{0}^{t}\left\|\partial_{x} F_{L}(\varphi, r)\right\|_{\mathrm{L}^{2}}^{2} \mathrm{~d} s\right\}^{1 / 2} \leq C L^{-1 / 2}\left(m_{1}+t\right)+C L^{-3 / 2} \sqrt{t} .
\end{aligned}
$$

In particular, $\left\|\partial_{x} \hat{\varphi}(t)\right\|_{\mathrm{L}^{2}} \leq C L^{-1 / 2}\left(m_{1}+t\right)$, which is the desired estimate. The bound on $\left\|\partial_{x}^{2} \hat{\varphi}\right\|_{L^{2}}$ is not sufficient yet, but replacing it into (5.18), we find $\int_{0}^{t}\left\|\partial_{x} \widetilde{F}_{L}(\varphi, r)\right\|_{\mathrm{L}^{2}}^{2} \mathrm{~d} s \leq$ $C L^{-1}\left(m_{1}^{2}+t^{2}\right)$, which in turn implies

$$
\left\|\partial_{x}^{2} \hat{\varphi}(t)\right\|_{\mathrm{L}^{2}} \leq \int_{0}^{t}\left\|\partial_{x}^{2} F_{L}(0,0)\right\|_{\mathrm{L}^{2}} \mathrm{~d} s+\left\{\int_{0}^{t}\left\|\partial_{x} \widetilde{F}_{L}(\varphi, r)\right\|_{\mathrm{L}^{2}}^{2} \mathrm{~d} s\right\}^{1 / 2} \leq C L^{-1 / 2}\left(m_{1}+t\right) .
$$

Thus, we have shown that $\|\hat{\varphi}\|_{\mathrm{H}_{L}^{2}}+\|\hat{r}\|_{\mathrm{H}_{L}^{1}} \leq C\left(m_{1}+t\right)$ for all $t \in[0, T]$, and the continuous dependence on the data follows by the same estimates. The proof of Lemma 5.2 is complete.

\section{$6 \quad$ Energy estimates}

This final section is devoted to the proof of Theorem 4.1 using energy estimates. Let $\eta_{+}, \eta_{-} \in\left(-\frac{1}{\sqrt{3}}, \frac{1}{\sqrt{3}}\right), \eta_{+} \neq \eta_{-}$, and assume that $(\psi, s) \in \mathcal{C}\left(\left[\tau_{1}, \tau_{2}\right], \mathrm{H}^{2}(\mathbb{R}) \times \mathrm{H}^{1}(\mathbb{R})\right)$ is a 
solution of (2.12) defined on some time interval $\left[\tau_{1}, \tau_{2}\right] \subset \mathbb{R}_{+}$and satisfying $\|\psi(\tau)\|_{\mathrm{H}^{2}}+$ $\|s(\tau)\|_{\mathrm{H}^{1}} \leq \varepsilon$ for all $\tau \in\left[\tau_{1}, \tau_{2}\right]$, for some sufficiently small $\varepsilon>0$ (to be specified later). We set $\left(\psi_{1}, s_{1}\right)=\left(\psi\left(\tau_{1}\right), s\left(\tau_{1}\right)\right)$. To control the evolution of the solutions $(\psi, s)$, we introduce the energy functionals

$$
\begin{aligned}
E_{0}(\tau) & =\frac{1}{2} \int_{\mathbb{R}} \psi(\tau, \xi)^{2} \mathrm{~d} \xi \\
E_{k+1}(\tau) & =\frac{1}{2} \int_{\mathbb{R}}\left(3 \delta^{(k)}(\tau, \xi)^{2}+s^{(k)}(\tau, \xi)^{2}\right) \mathrm{d} \xi, \quad k=0,1,2,
\end{aligned}
$$

where $\delta=\psi^{\prime}$.

The use of the quadratic forms $3\left(\delta^{(k)}\right)^{2}+\left(s^{(k)}\right)^{2}$ instead of $\left(\delta^{(k)}\right)^{2}+\left(s^{(k)}\right)^{2}$ in (6.1) can be understood as follows. For any $\beta>0$, the system (2.12), (2.14) for $\delta, s$ can be written in the form

$$
\left(\begin{array}{c}
\beta \dot{\delta} \\
\dot{s}
\end{array}\right)=\left(\begin{array}{cc}
a(\eta) & \beta \eta \\
\beta^{-1} a_{2}(\eta) & a_{1}(\eta)
\end{array}\right)\left(\begin{array}{c}
\beta \delta^{\prime \prime} \\
s^{\prime \prime}
\end{array}\right)+R_{\beta}\left(\xi, \tau ; \delta, \delta^{\prime}, s, s^{\prime}\right),
$$

where $a(\eta), a_{1}(\eta), a_{2}(\eta)$ are defined in (2.13) and the remainder $R_{\beta}$ contains only lower order derivatives of $\delta, s$. If $M_{\beta}(\eta)$ denotes the $2 \times 2$ matrix in the right-hand side of (6.2), then $\operatorname{Tr} M_{\beta}(\eta)=2$ and $\operatorname{Det} M_{\beta}(\eta)=1$ for all $\beta>0,|\eta|<1 / \sqrt{3}$, so that 1 is always a double eigenvalue of $M_{\beta}$. However, the evolution of the functionals $\int\left(\beta^{2}\left(\delta^{(k)}\right)^{2}+\left(s^{(k)}\right)^{2}\right) \mathrm{d} \xi$ is determined (up to lower order terms) by the symmetrized matrix $M_{\beta}^{s}=\frac{1}{2}\left(M_{\beta}+M_{\beta}^{\mathrm{t}}\right)$, which is not necessarily positive definite. Our choice of $E_{k+1}(\tau)$ in (6.1) is motivated by the fact that $M_{\beta}^{s}$ is positive definite for all $|\eta|<1 / \sqrt{3}$ if and only if $\beta=\sqrt{3}$. In this case, the eigenvalues are given by

$$
\mu_{ \pm}(\eta)=1 \pm \frac{\sqrt{3}|\eta|}{6\left(1-\eta^{2}\right)^{2}}\left(3-2 \eta^{2}+3 \eta^{4}\right)
$$

In the sequel, we set $\eta_{0}=\max \left(\left|\eta_{+}\right|,\left|\eta_{-}\right|\right)<1 / \sqrt{3}, \bar{\eta}=\frac{1}{2}\left(\eta_{0}+1 / \sqrt{3}\right), \bar{\mu}=\mu_{-}(\bar{\eta})$, $\bar{a}=a(\bar{\eta})=\left(1-3 \bar{\eta}^{2}\right) /\left(1-\bar{\eta}^{2}\right)$, and we assume that $\varepsilon \leq \bar{\eta}-\eta_{0}$. This implies in particular that $|\eta(\tau, \xi)|=|\widetilde{\eta}(\tau, \xi)+\delta(\tau, \xi)| \leq \eta_{0}+\varepsilon \leq \bar{\eta}<1 / \sqrt{3}$ for all $\xi \in \mathbb{R}, \tau \in\left[\tau_{1}, \tau_{2}\right]$.

\section{Lemma 6.1}

Under the assumptions above, we have $E_{0} \in \mathcal{C}^{1}\left(\left[\tau_{1}, \tau_{2}\right]\right)$ and, for all $\nu>0$, there exists a constant $K_{2}>0$ such that

$$
\dot{E}_{0}(\tau) \leq-\left(\frac{3}{2}-\nu\right) E_{0}(\tau)-\bar{a} \int \delta^{2} \mathrm{~d} \xi+K_{2} \int s^{\prime 2} \mathrm{~d} \xi
$$

for all $\tau \in\left[\tau_{1}, \tau_{2}\right]$, where $\bar{a}=a(\bar{\eta})=\left(1-3 \bar{\eta}^{2}\right) /\left(1-\bar{\eta}^{2}\right)>0$.

Remark. Here and in the sequel, $K_{2}, K_{3}, \ldots$ denote positive constants depending on $\eta_{+}, \eta_{-}$, but not on the interval $\left[\tau_{1}, \tau_{2}\right] \subset \mathbb{R}_{+}$nor on the solution $(\psi, s)$, provided $\|\psi(\tau)\|_{\mathrm{H}^{2}}+$ $\|s(\tau)\|_{\mathrm{H}^{1}} \leq \varepsilon$.

Proof: By hypothesis on $\psi$, we have $E_{0} \in \mathcal{C}\left(\left[\tau_{1}, \tau_{2}\right]\right.$. To prove the differentiability, we first assume that the initial data $\left(\psi_{1}, s_{1}\right)$ belong to $\mathcal{S}(\mathbb{R})$, the space of rapidly decreasing $\mathcal{C}^{\infty}$ functions on $\mathbb{R}$. Then $\psi(\tau), s(\tau) \in \mathcal{S}(\mathbb{R})$ for all $\tau \in\left[\tau_{1}, \tau_{2}\right], E_{0} \in \mathcal{C}^{\infty}\left(\left[\tau_{1}, \tau_{2}\right]\right)$, and a direct calculation shows that

$$
\dot{E}_{0}(\tau)=-\int \psi^{\prime}\left(A\left(\widetilde{\eta}+\psi^{\prime}\right)-A(\widetilde{\eta})\right) \mathrm{d} \xi-\frac{3}{4} \int \psi^{2} \mathrm{~d} \xi+\int \eta s^{\prime} \psi \mathrm{d} \xi
$$


In the general case, we use the fact that the solution $(\psi(\tau), s(\tau))$ depends continuously on the data $\left(\psi_{1}, s_{1}\right) \in \mathrm{H}^{2}(\mathbb{R}) \times \mathrm{H}^{1}(\mathbb{R})$, see Proposition 5.1. If $F(\tau)$ denotes the right-hand side of $(6.5)$, we see that (for fixed $\tau$ ) both $E_{0}(\tau)$ and $E_{0}\left(\tau_{1}\right)+\int_{\tau_{1}}^{\tau} F(t) \mathrm{d} t$ are continuous functions of $\left(\psi_{1}, s_{1}\right)$. Since these functions coincide on a dense subset (namely, $\mathcal{S}^{2}(\mathbb{R})$ ), they must be equal everywhere. This shows that $E_{0} \in \mathcal{C}^{1}\left(\left[\tau_{1}, \tau_{2}\right]\right)$ and satisfies $(6.5)$.

To prove (6.4), we first note that $A\left(\widetilde{\eta}+\psi^{\prime}\right)-A(\widetilde{\eta})=a(\hat{\eta}) \psi^{\prime}$ for some $\hat{\eta} \in\left[\widetilde{\eta}, \widetilde{\eta}+\psi^{\prime}\right]$. Since $|\hat{\eta}| \leq \bar{\eta}$ by assumption, we have $\psi^{\prime}\left(A\left(\widetilde{\eta}+\psi^{\prime}\right)-A(\widetilde{\eta})\right)=a(\hat{\eta}) \psi^{\prime 2} \geq \bar{a} \psi^{\prime 2}$. On the other hand, for all $\nu>0$, one has $\left|\eta s^{\prime} \psi\right| \leq\left|s^{\prime} \psi\right| \leq \frac{\nu}{2} \psi^{2}+\frac{1}{2 \nu} s^{\prime 2}$. We thus find

$$
\dot{E}_{0}(\tau) \leq-\bar{a} \int \psi^{\prime 2} \mathrm{~d} \xi-\left(\frac{3}{4}-\frac{\nu}{2}\right) \int \psi^{2} \mathrm{~d} \xi+K_{2} \int s^{\prime 2} \mathrm{~d} \xi
$$

where $K_{2}=(2 \nu)^{-1}$. This concludes the proof of Lemma 6.1.

\section{Lemma 6.2}

Under the same assumptions, we have $E_{1} \in \mathcal{C}^{1}\left(\left[\tau_{1}, \tau_{2}\right]\right)$, and there exists a constant $K_{3}>0$ such that

$$
\dot{E}_{1}(\tau) \leq-\bar{\mu} E_{2}(\tau)-\frac{1}{2} \mathrm{e}^{\tau} \int s^{2} \mathrm{~d} \xi+K_{3}\left(\mathrm{e}^{-\tau}+\int\left(s^{2}+\delta^{2}\right) \mathrm{d} \xi\right)
$$

for all $\tau \in\left[\tau_{1}, \tau_{2}\right]$, where $\bar{\mu}=\mu_{-}(\bar{\eta})$ is given by $(6.3)$.

Proof: As in the proof of Lemma 6.1, the differentiability of $\int \delta^{2} \mathrm{~d} \xi$ and $\int s^{2} \mathrm{~d} \xi$ is easily verified using a density argument. From (2.14), we have

$$
\begin{aligned}
\int \delta \dot{\delta} \mathrm{d} \xi & =-\int \delta^{\prime}(A(\widetilde{\eta}+\delta)-A(\widetilde{\eta}))^{\prime} \mathrm{d} \xi-\frac{1}{4} \int \delta^{2} \mathrm{~d} \xi-\int \eta s^{\prime} \delta^{\prime} \mathrm{d} \xi \\
& =-\int\left(a(\eta) \delta^{\prime 2}+\eta s^{\prime} \delta^{\prime}\right) \mathrm{d} \xi-\frac{1}{4} \int \delta^{2} \mathrm{~d} \xi-\int \widetilde{\eta}^{\prime} \delta^{\prime}(a(\widetilde{\eta}+\delta)-a(\widetilde{\eta})) \mathrm{d} \xi
\end{aligned}
$$

Since $\left|\widetilde{\eta}^{\prime} \delta^{\prime}(a(\widetilde{\eta}+\delta)-a(\widetilde{\eta}))\right| \leq\left|\widetilde{\eta}^{\prime}\right|\left|a^{\prime}(\bar{\eta})\right|\left|\delta \delta^{\prime}\right| \leq \frac{\bar{\mu}}{4} \delta^{2}+C_{1} \delta^{2}$ for some $C_{1}>0$, we obtain

$$
\int \delta \dot{\delta} \mathrm{d} \xi \leq-\int\left(a(\eta) \delta^{\prime 2}+\eta s^{\prime} \delta^{\prime}\right) \mathrm{d} \xi+\frac{\bar{\mu}}{4} \int \delta^{\prime 2} \mathrm{~d} \xi+C_{1} \int \delta^{2} \mathrm{~d} \xi
$$

On the other hand, using (2.12) and integrating by parts, we find

$$
\begin{aligned}
\int s \dot{s} \mathrm{~d} \xi= & -\int\left(a_{1}(\eta) s^{2}+a_{2}(\eta) s^{\prime} \delta^{\prime}\right) \mathrm{d} \xi-\frac{1}{4} \int s^{2} \mathrm{~d} \xi-2 \mathrm{e}^{\tau} \int\left(1-\eta^{2}\right) s\left(\mathrm{e}^{s}-1\right) \mathrm{d} \xi \\
& +\int\left(\frac{1}{2} s s^{2}-a_{1}^{\prime}(\eta) \eta^{\prime} s s^{\prime}+a_{2}(\eta) \widetilde{\eta}^{\prime \prime} s-a_{2}^{\prime}(\eta) \eta^{\prime} \delta^{\prime} s+a_{3}(\eta) s \eta^{\prime 2}\right) \mathrm{d} \xi
\end{aligned}
$$

Since $\eta^{2} \leq 1 / 3$ and $|s| \leq \varepsilon \leq 1 / 2$, we have $2\left(1-\eta^{2}\right) s\left(\mathrm{e}^{s}-1\right) \geq s^{2}$. The other terms are bounded as follows: $\left|s s^{\prime 2}\right| \leq \varepsilon s^{\prime 2},\left|a_{1}^{\prime}(\eta) \eta^{\prime} s s^{\prime}\right| \leq C\left|\left(\widetilde{\eta}^{\prime}+\delta^{\prime}\right) s s^{\prime}\right| \leq C\left(\left|s s^{\prime}\right|+\varepsilon\left|s^{\prime} \delta^{\prime}\right|\right)$, $\left|a_{2}(\eta) \widetilde{\eta}^{\prime \prime} s\right| \leq C|s| \mathrm{e}^{-|\xi|},\left|a_{2}^{\prime}(\eta) \eta^{\prime} \delta^{\prime} s\right| \leq C\left(\left|s \delta^{\prime}\right|+\varepsilon{\delta^{\prime}}^{2}\right),\left|a_{3}(\eta) s \eta^{\prime 2}\right| \leq C\left(|s| \mathrm{e}^{-|\xi|}+\varepsilon \delta^{\prime 2}\right)$. We thus obtain

$$
\begin{aligned}
\int s \dot{s} \mathrm{~d} \xi \leq & -\int\left(a_{1}(\eta){s^{\prime}}^{2}+a_{2}(\eta) s^{\prime} \delta^{\prime}\right) \mathrm{d} \xi-\left(\frac{1}{4}+\mathrm{e}^{\tau}\right) \int s^{2} \mathrm{~d} \xi \\
& +C_{2} \int\left(|s| \mathrm{e}^{-|\xi|}+\left|s s^{\prime}\right|+\left|s \delta^{\prime}\right|+\varepsilon\left(s^{\prime 2}+\delta^{\prime 2}\right)\right) \mathrm{d} \xi
\end{aligned}
$$


for some $C_{2}>0$. Now, assuming that $\varepsilon \leq \bar{\mu} /\left(4 C_{2}\right)$, we have $C_{2} \varepsilon\left(s^{\prime 2}+\delta^{\prime 2}\right) \leq \bar{\mu}\left(s^{\prime 2}+\delta^{\prime 2}\right) / 4$. In addition, $C_{2}|s| \mathrm{e}^{-|\xi|} \leq \frac{1}{2} s^{2} \mathrm{e}^{\tau}+C \mathrm{e}^{-\tau} \mathrm{e}^{-2|\xi|}$ and $C_{2}\left(\left|s s^{\prime}\right|+\left|s \delta^{\prime}\right|\right) \leq \bar{\mu}\left(s^{\prime 2}+{\delta^{\prime}}^{2}\right) / 4+C s^{2}$. Therefore,

$$
\begin{aligned}
\int s \dot{s} \mathrm{~d} \xi= & -\int\left(a_{1}(\eta){s^{\prime}}^{2}+a_{2}(\eta) s^{\prime} \delta^{\prime}\right) \mathrm{d} \xi+\left(C_{3}-\frac{1}{2} \mathrm{e}^{\tau}\right) \int s^{2} \mathrm{~d} \xi \\
& +\frac{\mu}{2} \int\left(s^{\prime 2}+\delta^{\prime 2}\right) \mathrm{d} \xi+C_{3} \mathrm{e}^{-\tau},
\end{aligned}
$$

for some $C_{3}>0$.

Finally, by definition of $\mu_{-}(\eta)$ (see (6.3)), we have the inequality

$$
\int\left(3 a(\eta) \delta^{\prime 2}+\left(3 \eta+a_{2}(\eta)\right) s^{\prime} \delta^{\prime}+a_{1}(\eta) s^{\prime 2}\right) \mathrm{d} \xi \geq 2 \mu_{-}(\bar{\eta}) E_{2}=2 \bar{\mu} E_{2} .
$$

Thus, multiplying (6.6) by 3 and adding the result to (6.7), we obtain

$$
\dot{E}_{1}(\tau) \leq-\bar{\mu} E_{2}(\tau)+\left(C_{3}-\frac{1}{2} \mathrm{e}^{\tau}\right) \int s^{2} \mathrm{~d} \xi+3 C_{1} \int \delta^{2} \mathrm{~d} \xi+C_{3} \mathrm{e}^{-\tau}
$$

for all $\tau \in\left[\tau_{1}, \tau_{2}\right]$. This concludes the proof of Lemma 6.2.

\section{Lemma 6.3}

Under the same assumptions, $(\psi, s) \in \mathrm{L}^{2}\left(\left[\tau_{1}, \tau_{2}\right], \mathrm{H}^{3}(\mathbb{R}) \times \mathrm{H}^{2}(\mathbb{R})\right), E_{2} \in \mathrm{W}^{1,1}\left(\left[\tau_{1}, \tau_{2}\right]\right)$, and there exists a constant $K_{4}>0$ such that

$$
\dot{E}_{2}(\tau) \leq-\bar{\mu} E_{3}(\tau)-\frac{1}{2} \mathrm{e}^{\tau} \int s^{\prime 2} \mathrm{~d} \xi+K_{4}\left(\mathrm{e}^{-\tau}+\mathrm{e}^{\tau} \int s^{2} \mathrm{~d} \xi+\int\left(s^{\prime 2}+\delta^{2}+\delta^{\prime 2}\right) \mathrm{d} \xi\right) .
$$

Remark. Due to the regularization, one also has $(\psi, s) \in \mathcal{C}\left(\left(\tau_{1}, \tau_{2}\right], \mathrm{H}^{3}(\mathbb{R}) \times \mathrm{H}^{2}(\mathbb{R})\right), E_{2} \in$ $\mathcal{C}^{1}\left(\left(\tau_{1}, \tau_{2}\right]\right)$, and $(6.9)$ holds for all $\tau \in\left(\tau_{1}, \tau_{2}\right]$.

Proof: As in the proof of Lemma 6.1, we first assume that $\psi(\tau), s(\tau) \in \mathcal{S}(\mathbb{R})$ for all $\tau \in\left[\tau_{1}, \tau_{2}\right]$. Then, we have from (2.14)

$$
\int \delta^{\prime} \dot{\delta}^{\prime} \mathrm{d} \xi=-\int \delta^{\prime \prime} \dot{\delta} \mathrm{d} \xi=-\int \delta^{\prime \prime}(A(\widetilde{\eta}+\delta)-A(\widetilde{\eta}))^{\prime \prime} \mathrm{d} \xi+\frac{1}{4} \int \delta^{\prime 2} \mathrm{~d} \xi-\int \delta^{\prime \prime}\left(\eta s^{\prime}\right)^{\prime} \mathrm{d} \xi
$$

Since $(A(\widetilde{\eta}+\delta)-A(\widetilde{\eta}))^{\prime \prime}=a(\eta) \delta^{\prime \prime}+(a(\widetilde{\eta}+\delta)-a(\widetilde{\eta})) \widetilde{\eta}^{\prime \prime}+\left(a^{\prime}(\widetilde{\eta}+\delta)-a^{\prime}(\widetilde{\eta})\right)\left(\widetilde{\eta}^{\prime}\right)^{2}+a^{\prime}(\eta)\left(2 \widetilde{\eta}^{\prime}+\right.$ $\left.\delta^{\prime}\right) \delta^{\prime}$ and $\left(\eta s^{\prime}\right)^{\prime}=\eta s^{\prime \prime}+\left(\widetilde{\eta}^{\prime}+\delta^{\prime}\right) s^{\prime}$, we obtain using straightforward bounds

$$
\begin{aligned}
\int \delta^{\prime} \dot{\delta}^{\prime} \mathrm{d} \xi \leq & -\int\left(a(\eta) \delta^{\prime \prime 2}+\eta s^{\prime \prime} \delta^{\prime \prime}\right) \mathrm{d} \xi+\frac{1}{4} \int \delta^{\prime 2} \mathrm{~d} \xi \\
& +C_{4} \int\left|\delta^{\prime \prime}\right|\left(|\delta|+\left|\delta^{\prime}\right|+\left|s^{\prime}\right|+\left|\delta^{\prime 2}\right|+\left|s^{\prime} \delta^{\prime}\right|\right) \mathrm{d} \xi
\end{aligned}
$$

for some $C_{4}>0$. Now, $C_{4}\left|\delta^{\prime \prime}\right|\left(|\delta|+\left|\delta^{\prime}\right|+\left|s^{\prime}\right|\right) \leq \frac{\bar{\mu}}{8} \delta^{\prime \prime 2}+C\left(\delta^{2}+\delta^{\prime 2}+s^{\prime 2}\right)$ and

$$
\begin{aligned}
C_{4}\left\|\delta^{\prime \prime} \delta^{\prime}\left(\left|\delta^{\prime}\right|+\left|s^{\prime}\right|\right)\right\|_{\mathrm{L}^{1}} & \leq C_{4}\left\|\delta^{\prime \prime}\right\|_{\mathrm{L}^{2}}^{3 / 2}\left\|\delta^{\prime}\right\|_{\mathrm{L}^{2}}^{1 / 2}\left(\left\|\delta^{\prime}\right\|_{\mathrm{L}^{2}}+\left\|s^{\prime}\right\|_{\mathrm{L}^{2}}\right) \leq 2 \varepsilon C_{4}\left\|\delta^{\prime \prime}\right\|_{\mathrm{L}^{2}}^{3 / 2}\left\|\delta^{\prime}\right\|_{\mathrm{L}^{2}}^{1 / 2} \\
& \leq \frac{\bar{\mu}}{8}\left\|\delta^{\prime \prime}\right\|_{\mathrm{L}^{2}}^{2}+C \varepsilon^{4}\left\|\delta^{\prime}\right\|_{\mathrm{L}^{2}}^{2}
\end{aligned}
$$

hence

$$
\int \delta^{\prime} \dot{\delta}^{\prime} \mathrm{d} \xi \leq-\int\left(a(\eta) \delta^{\prime \prime 2}+\eta s^{\prime \prime} \delta^{\prime \prime}\right) \mathrm{d} \xi+\frac{\bar{\mu}}{4} \int \delta^{\prime \prime 2} \mathrm{~d} \xi+C_{5} \int\left(\delta^{2}+\delta^{2}+s^{2}\right) \mathrm{d} \xi
$$


for some $C_{5}>0$.

On the other hand, assuming always $\psi(\tau), s(\tau) \in \mathcal{S}(\mathbb{R})$, we have from (2.12)

$$
\begin{aligned}
\int s^{\prime} \dot{s}^{\prime} \mathrm{d} \xi= & -\int s^{\prime \prime} \dot{s} \mathrm{~d} \xi=-\int\left(a_{1}(\eta) s^{\prime \prime 2}+a_{2}(\eta) s^{\prime \prime} \eta^{\prime \prime}\right) \mathrm{d} \xi \\
& +\frac{1}{4} \int s^{\prime 2} \mathrm{~d} \xi+2 \mathrm{e}^{\tau} \int s^{\prime \prime}\left(1-\eta^{2}\right)\left(\mathrm{e}^{s}-1\right) \mathrm{d} \xi-\int a_{3}(\eta) s^{\prime \prime} \eta^{\prime 2} \mathrm{~d} \xi .
\end{aligned}
$$

Integrating by parts, we obtain

$$
\begin{aligned}
& -\int a_{2}(\eta) s^{\prime \prime} \widetilde{\eta}^{\prime \prime} \mathrm{d} \xi=\int s^{\prime}\left(a_{2}(\eta) \widetilde{\eta}^{\prime \prime \prime}+a_{2}^{\prime}(\eta) \widetilde{\eta}^{\prime \prime}\left(\widetilde{\eta}^{\prime}+\delta^{\prime}\right)\right) \mathrm{d} \xi \leq C \int\left|s^{\prime}\right|\left(\mathrm{e}^{-|\xi|}+\left|\delta^{\prime}\right|\right) \mathrm{d} \xi, \\
& -\int a_{3}(\eta) s^{\prime \prime} \eta^{\prime 2} \mathrm{~d} \xi=\int s^{\prime}\left(a_{3}^{\prime}(\eta) \eta^{\prime 3}+2 a_{3}(\eta) \eta^{\prime} \eta^{\prime \prime}\right) \mathrm{d} \xi \\
& \leq C \int\left|s^{\prime}\right|\left(\mathrm{e}^{-|\xi|}+\left|\delta^{\prime}\right|+\left|\delta^{\prime \prime}\right|+\left|\delta^{\prime 3}\right|+\left|\delta^{\prime} \delta^{\prime \prime}\right|\right) \mathrm{d} \xi, \\
& 2 \mathrm{e}^{\tau} \int s^{\prime \prime}\left(1-\eta^{2}\right)\left(\mathrm{e}^{s}-1\right) \mathrm{d} \xi=-2 \mathrm{e}^{\tau} \int\left(1-\eta^{2}\right) \mathrm{e}^{s} s^{\prime 2} \mathrm{~d} \xi+4 \mathrm{e}^{\tau} \int \eta \eta^{\prime}\left(\mathrm{e}^{s}-1\right) s^{\prime} \mathrm{d} \xi \\
& \leq-\mathrm{e}^{\tau} \int s^{\prime 2} \mathrm{~d} \xi+C \mathrm{e}^{\tau} \int\left|s s^{\prime}\right|\left(1+\left|\delta^{\prime}\right|\right) \mathrm{d} \xi .
\end{aligned}
$$

Therefore,

$$
\int s^{\prime} \dot{s}^{\prime} \mathrm{d} \xi=-\int\left(a_{1}(\eta) s^{\prime \prime 2}+a_{2}(\eta) s^{\prime \prime} \delta^{\prime \prime}\right) \mathrm{d} \xi+\left(\frac{1}{4}-\mathrm{e}^{\tau}\right) \int s^{\prime 2} \mathrm{~d} \xi+\int R \mathrm{~d} \xi,
$$

where $|R| \leq C_{6}\left|s^{\prime}\right|\left(\mathrm{e}^{-|\xi|}+\left|\delta^{\prime}\right|+\left|\delta^{\prime \prime}\right|+\left|\delta^{\prime 3}\right|+\left|\delta^{\prime} \delta^{\prime \prime}\right|\right)+C_{6} \mathrm{e}^{\tau}\left|s s^{\prime}\right|\left(1+\left|\delta^{\prime}\right|\right)$ for some $C_{6}>0$. Using the bounds $C_{6}\left|s^{\prime}\right| \mathrm{e}^{-|\xi|} \leq \frac{1}{4} \mathrm{e}^{\tau} s^{\prime 2}+C \mathrm{e}^{-\tau} \mathrm{e}^{-2|\xi|}$ and

$$
\begin{gathered}
C_{6}\left\|s^{\prime}\left(\left|\delta^{\prime}\right|+\left|\delta^{\prime \prime}\right|+\left|\delta^{\prime 3}\right|+\left|\delta^{\prime} \delta^{\prime \prime}\right|\right)\right\|_{\mathrm{L}^{1}} \leq \frac{\bar{\mu}}{2}\left\|\delta^{\prime \prime}\right\|_{\mathrm{L}^{2}}^{2}+C\left(\left\|s^{\prime}\right\|_{\mathrm{L}^{2}}^{2}+\left\|\delta^{\prime}\right\|_{\mathrm{L}^{2}}^{2}\right), \\
C_{6} \mathrm{e}^{\tau}\left\|s s^{\prime}\left(1+\left|\delta^{\prime}\right|\right)\right\|_{\mathrm{L}^{1}} \leq \frac{1}{4} \mathrm{e}^{\tau}\left\|s^{\prime}\right\|_{\mathrm{L}^{2}}^{2}+C \mathrm{e}^{\tau}\|s\|_{\mathrm{L}^{2}}^{2},
\end{gathered}
$$

we find

$$
\begin{aligned}
\int s^{\prime} \dot{s}^{\prime} \mathrm{d} \xi \leq & -\int\left(a_{1}(\eta) s^{\prime \prime 2}+a_{2}(\eta) s^{\prime \prime} \delta^{\prime \prime}\right) \mathrm{d} \xi+\left(C_{7}-\frac{1}{2} \mathrm{e}^{\tau}\right) \int s^{\prime 2} \mathrm{~d} \xi \\
& +\frac{\mu}{2} \int \delta^{\prime \prime 2} \mathrm{~d} \xi+C_{7}\left(\mathrm{e}^{-\tau}+\int\left(\mathrm{e}^{\tau} s^{2}+\delta^{\prime 2}\right) \mathrm{d} \xi\right)
\end{aligned}
$$

for some $C_{7}>0$. Finally, combining (6.11), (6.13) and using the analogue of (6.8), we arrive at (6.9). This proves the claim when $\psi(\tau), s(\tau) \in \mathcal{S}(\mathbb{R})$.

To infer the general case, we use again a density argument. Integrating (6.9), we obtain

$$
E_{2}\left(\tau_{2}\right)+\bar{\mu} \int_{\tau_{1}}^{\tau_{2}} E_{3}(\tau) \mathrm{d} \tau \leq E_{2}\left(\tau_{1}\right)+\int_{\tau_{1}}^{\tau_{2}} F(\tau) \mathrm{d} \tau
$$

where $F(\tau) \leq C\left(\|\psi(\tau)\|_{\mathrm{H}^{2}}^{2}+\|s(\tau)\|_{\mathrm{H}^{1}}^{2}\right)$ for all $\tau \in\left[\tau_{1}, \tau_{2}\right]$. Since $\mathcal{S}^{2}(\mathbb{R})$ is dense in $\mathrm{H}^{2}(\mathbb{R}) \times \mathrm{H}^{1}(\mathbb{R})$, the estimate (6.14) shows that, if $(\psi, s) \in \mathcal{C}\left(\left[\tau_{1}, \tau_{2}\right], \mathrm{H}^{2}(\mathbb{R}) \times \mathrm{H}^{1}(\mathbb{R})\right)$ is any solution satisfying the usual assumptions, then $(\psi, s) \in \mathrm{L}^{2}\left(\left[\tau_{1}, \tau_{2}\right], \mathrm{H}^{3}(\mathbb{R}) \times \mathrm{H}^{2}(\mathbb{R})\right)$. Using this result, it follows immediately from (6.10), (6.12) that $\dot{E}_{2} \in \mathrm{L}^{1}\left(\left[\tau_{1}, \tau_{2}\right]\right)$, hence $E_{2} \in \mathrm{W}^{1,1}\left(\left[\tau_{1}, \tau_{2}\right]\right)$, and the same calculations as above show that (6.9) holds in the general case. This concludes the proof of Lemma 6.3.

Aa a consequence of Lemmas 6.1, 6.2, 6.3, we have the following estimate: 


\section{Proposition 6.4}

There exist $\varepsilon_{2}>0, K_{5} \geq 1$ such that, if $(\psi, s) \in C\left(\left[\tau_{1}, \tau_{2}\right], \mathrm{H}^{2}(\mathbb{R}) \times \mathrm{H}^{1}(\mathbb{R})\right)$ is any solution of (2.12) satisfying $\|\psi(\tau)\|_{\mathrm{H}^{2}}+\|s(\tau)\|_{\mathrm{H}^{1}} \leq \varepsilon_{2}$ for all $\tau \in\left[\tau_{1}, \tau_{2}\right]$, then

$$
\|\psi(\tau)\|_{\mathrm{H}^{2}}+\|s(\tau)\|_{\mathrm{H}^{1}} \leq K_{5} \mathrm{e}^{-\tau / 2}\left(1+\mathrm{e}^{\tau_{1} / 2}\left(\left\|\psi\left(\tau_{1}\right)\right\|_{\mathrm{H}^{2}}+\left\|s\left(\tau_{1}\right)\right\|_{\mathrm{H}^{1}}\right)\right),
$$

for all $\tau \in\left[\tau_{1}, \tau_{2}\right]$.

Proof: Fix $\gamma \in(1 / 2,3 / 4)$ and let $\nu=3 / 2-2 \gamma$. We set $\varepsilon_{2}=\min \left(\bar{\eta}-\eta_{0}, \bar{\mu} /\left(4 C_{2}\right)\right)$, where $C_{2}$ is defined in the proof of Lemma 6.2. Then, for $A, B>0$ large enough, we define

$$
G_{2}(\tau)=B\left(A E_{0}(\tau)+E_{1}(\tau)\right)+E_{2}(\tau), \quad \tau \in\left[\tau_{1}, \tau_{2}\right]
$$

For instance, we may choose $\bar{a} A=K_{3}+11 / 4$ and assume that $B \geq 2 K_{4}+1,3 \bar{\mu} B \geq$ $2 K_{4}+9 / 2$. Then, using Lemmas 6.1, 6.2, 6.3, we find

$$
\dot{G}_{2}(\tau) \leq-2 \gamma G_{2}(\tau)+\frac{1}{2}\left(C_{8}-\mathrm{e}^{\tau}\right) \int\left(s^{2}+s^{\prime 2}\right) \mathrm{d} \xi+C_{8} \mathrm{e}^{-\tau}, \quad \tau \in\left[\tau_{1}, \tau_{2}\right]
$$

for some $C_{8}>0$. Integrating this inequality, we easily obtain

$$
G_{2}(\tau) \leq C_{9}\left(G_{2}\left(\tau_{1}\right) \mathrm{e}^{-2 \gamma\left(\tau-\tau_{1}\right)}+\mathrm{e}^{-\tau}\right), \quad \tau \in\left[\tau_{1}, \tau_{2}\right]
$$

for some $C_{9}>0$. Since $2 \gamma>1$ and $G_{2}(\tau)$ is equivalent to $\|\psi(\tau)\|_{\mathrm{H}^{2}}^{2}+\|s(\tau)\|_{\mathrm{H}^{1}}^{2}$, this proves (6.15).

Combining Proposition 5.1 and Proposition 6.4, we are now able to complete the proof of Theorem 4.1.

Proof of Theorem 4.1. Fix $\gamma \in(1 / 2,3 / 4)$, and let $\varepsilon_{3}=\min \left(\varepsilon_{1}, \varepsilon_{2}\right)$, where $\varepsilon_{1}$ is defined in Proposition 5.1 and $\varepsilon_{2}$ in Proposition 6.4. We set

$$
\varepsilon_{0}=\frac{\varepsilon_{3}}{4 K_{5}}, \quad \tau_{0}=\max \left(0,-2 \log \frac{\varepsilon_{3}}{4 K_{5}}\right)
$$

where $K_{5}$ is defined in Proposition 6.4. Then, for all $\left(\psi_{0}, s_{0}\right) \in \mathrm{H}^{2}(\mathbb{R}) \times \mathrm{H}^{1}(\mathbb{R})$ satisfying $\left\|\psi_{0}\right\|_{\mathrm{H}^{2}}+\left\|s_{0}\right\|_{\mathrm{H}^{1}} \leq \varepsilon_{0}$, the system (2.12) has a unique global solution $(\psi, s) \in$ $\mathcal{C}\left(\left[\tau_{0}, \infty\right), \mathrm{H}^{2}(\mathbb{R}) \times \mathrm{H}^{1}(\mathbb{R})\right)$ with initial data $\left(\psi\left(\tau_{0}\right), s\left(\tau_{0}\right)\right)=\left(\psi_{0}, s_{0}\right)$. Indeed, according to our local existence result (Proposition 5.1), it suffices to show that the solution $(\psi(\tau), s(\tau))$ satisfies $\|\psi(\tau)\|_{\mathrm{H}^{2}}+\|s(\tau)\|_{\mathrm{H}^{1}}<\varepsilon_{3}$ whenever it exists. Assume on the contrary that there exists a time $\tau_{3}>\tau_{0}$ such that $\left\|\psi\left(\tau_{3}\right)\right\|_{\mathrm{H}^{2}}+\left\|s\left(\tau_{3}\right)\right\|_{\mathrm{H}^{1}}=\varepsilon_{3}$ and $\|\psi(\tau)\|_{\mathrm{H}^{2}}+\|s(\tau)\|_{\mathrm{H}^{1}}<\varepsilon_{3}$ for all $\tau \in\left[\tau_{0}, \tau_{3}\right)$. Then, according to Proposition 6.4, we have

$$
\left\|\psi\left(\tau_{3}\right)\right\|_{\mathrm{H}^{2}}+\left\|s\left(\tau_{3}\right)\right\|_{\mathrm{H}^{1}} \leq K_{5} \mathrm{e}^{-\tau_{3} / 2}\left(1+\varepsilon_{0} \mathrm{e}^{\tau_{0} / 2}\right) \leq K_{5}\left(\mathrm{e}^{-\tau_{0} / 2}+\varepsilon_{0}\right) \leq \frac{\varepsilon_{3}}{2}
$$

which is a contradiction. Therefore, $(\psi(\tau), s(\tau))$ exists for all $\tau \geq \tau_{0}$ and satisfies $\|\psi(\tau)\|_{\mathrm{H}^{2}}+\|s(\tau)\|_{\mathrm{H}^{1}} \leq \varepsilon_{3} / 2$. In particular, we have from (6.15)

$$
\|\psi(\tau)\|_{\mathrm{H}^{2}}+\|s(\tau)\|_{\mathrm{H}^{1}} \leq C \mathrm{e}^{-\tau / 2}, \quad \tau \geq \tau_{0}
$$


for some $C>0$. It remains to show that $\|\psi(\tau)\|_{\mathrm{H}^{2}}+\|s(\tau)\|_{\mathrm{H}^{1}}=\mathcal{O}\left(\mathrm{e}^{-\gamma \tau}\right)$ as $\tau \rightarrow+\infty$. In fact, we shall prove that $\|\psi(\tau)\|_{\mathrm{H}^{2}}=\mathcal{O}\left(\mathrm{e}^{-\gamma \tau}\right)$ and $\|s(\tau)\|_{\mathrm{H}^{1}}=\mathcal{O}\left(\mathrm{e}^{-\tau}\right)$ as $\tau \rightarrow+\infty$.

We begin with $\|s(\tau)\|_{\mathrm{L}^{2}}$. From (6.7) and (6.16), we have for all $\tau \geq \tau_{0}$ :

$$
\frac{d}{d \tau} \int s^{2} \mathrm{~d} \xi \leq\left(\bar{C}_{1}-\mathrm{e}^{\tau}\right) \int s^{2} \mathrm{~d} \xi+\bar{C}_{1}\left(\mathrm{e}^{-\tau}+\int \delta^{\prime 2} \mathrm{~d} \xi\right) \leq-\mathrm{e}^{\tau} \int s^{2} \mathrm{~d} \xi+\bar{C}_{2} \mathrm{e}^{-\tau}
$$

for some $\bar{C}_{1}, \bar{C}_{2}>0$. This differential inequality implies that $\|s(\tau)\|_{\mathrm{L}^{2}}=\mathcal{O}\left(\mathrm{e}^{-\tau}\right)$ as $\tau \rightarrow+\infty$. Indeed, let $f(\tau)=\mathrm{e}^{2 \tau}\|s(\tau)\|_{\mathrm{L}^{2}}^{2}$, and assume that $\mathrm{e}^{\tau} \geq 4$. Then $\dot{f}(\tau) \leq$ $2 f(\tau)+\mathrm{e}^{\tau}\left(\bar{C}_{2}-f(\tau)\right) \leq e^{\tau}\left(\bar{C}_{2}-f(\tau) / 2\right)$, hence $f(\tau) \leq 2 \bar{C}_{2}+1$ if $\tau$ is sufficiently large.

To prove that $\left\|s^{\prime}(\tau)\right\|_{\mathrm{L}^{2}}=\mathcal{O}\left(\mathrm{e}^{-\tau}\right)$ as $\tau \rightarrow+\infty$, we first have to show that $E_{3}(\tau)=$ $\mathcal{O}\left(\mathrm{e}^{-\tau}\right)$, see (6.1). This estimate can be established using exactly the same techniques as above. Indeed, due to the parabolic regularization, $E_{3}(\tau)$ is continuously differentiable for all $\tau>\tau_{0}$. Thus, proceeding as in Lemma 6.2 or 6.3, one verifies that there exists a $K_{6}>0$ such that

$$
\begin{aligned}
\dot{E}_{3}(\tau) \leq & -\frac{1}{2} \mathrm{e}^{\tau} \int s^{\prime \prime 2} \mathrm{~d} \xi+K_{6} \int\left(s^{\prime \prime 2}+\delta^{2}+\delta^{\prime 2}+\delta^{\prime \prime 2}\right) \mathrm{d} \xi \\
& +K_{6}\left(\mathrm{e}^{-\tau}+\mathrm{e}^{\tau} \int\left(s^{2}+s^{\prime 2}\right) \mathrm{d} \xi\right),
\end{aligned}
$$

for all $\tau \geq \tau_{0}+1$ (say). Then, defining $G_{3}(\tau)=D G_{2}(\tau)+E_{3}(\tau)$ for some sufficiently large $D>0$ and proceeding as in Proposition 6.4, we obtain

$$
\dot{G}_{3}(\tau) \leq-2 \gamma G_{3}(\tau)-\frac{1}{2} \mathrm{e}^{\tau} \int\left(s^{2}+s^{\prime 2}+s^{\prime \prime 2}\right) \mathrm{d} \xi+K_{7} \mathrm{e}^{-\tau}
$$

for some $K_{7}>0$. By Gronwall's lemma, we have

$$
G_{3}(\tau)+\frac{1}{2} \int_{\tau_{0}+1}^{\tau} \mathrm{e}^{-2 \gamma(\tau-t)} \mathrm{e}^{t}\|s(t)\|_{\mathrm{H}^{2}}^{2} \mathrm{~d} t \leq \bar{C}_{3} \mathrm{e}^{-\tau}, \quad \tau \geq \tau_{0}+1,
$$

for some $\bar{C}_{3}>0$. In particular, $E_{3}(\tau)=\mathcal{O}\left(\mathrm{e}^{-\tau}\right)$ as $\tau \rightarrow+\infty$. Using this result, we deduce from 6.13 )

$$
\begin{aligned}
\frac{d}{d \tau} \int s^{\prime 2} \mathrm{~d} \xi & \leq\left(\bar{C}_{4}-\mathrm{e}^{\tau}\right) \int{s^{\prime}}^{2} \mathrm{~d} \xi+\bar{C}_{4}\left(\mathrm{e}^{-\tau}+\int\left(\mathrm{e}^{\tau} s^{2}+{\delta^{\prime}}^{2}+\delta^{\prime \prime 2}\right) \mathrm{d} \xi\right) \\
& \leq\left(\bar{C}_{4}-\mathrm{e}^{\tau}\right) \int s^{\prime 2} \mathrm{~d} \xi+\bar{C}_{5} \mathrm{e}^{-\tau}
\end{aligned}
$$

hence $\left\|s^{\prime}(\tau)\right\|_{\mathrm{L}^{2}}=\mathcal{O}\left(\mathrm{e}^{-\tau}\right)$ as $\tau \rightarrow+\infty$.

Finally, choosing $A, B>0$ large enough, we define

$$
H_{2}(\tau)=B\left(A \int \psi^{2} \mathrm{~d} \xi+\int \delta^{2} \mathrm{~d} \xi\right)+\int \delta^{\prime 2} \mathrm{~d} \xi
$$

Using (6.4), (6.6), (6.11) and proceeding as in the proof of Proposition 6.4, we obtain

$$
\dot{H}_{2}(\tau) \leq-2 \gamma H_{2}(\tau)+\bar{C}_{6}\|s(\tau)\|_{\mathrm{H}^{2}}^{2},
$$

for some $\bar{C}_{6}>0$. To control the last term in the right-hand side, we recall that

$$
\int_{\tau_{0}+1}^{\tau} \mathrm{e}^{(2 \gamma+1) t}\|s(t)\|_{\mathrm{H}^{2}}^{2} \mathrm{~d} t \leq 2 \bar{C}_{3} \mathrm{e}^{(2 \gamma-1) \tau}, \quad \tau \geq \tau_{0}+1
$$


by (6.17). Since $2 \gamma+1>2$, it follows that $\int_{\tau_{0}}^{\infty} \mathrm{e}^{\lambda \tau}\|s(\tau)\|_{\mathrm{H}^{2}}^{2} \mathrm{~d} \tau<\infty$ for all $\lambda<2$. Therefore, we conclude from (6.18) that

$$
H_{2}(\tau) \leq \mathrm{e}^{-2 \gamma\left(\tau-\tau_{0}\right)} H_{2}\left(\tau_{0}\right)+\bar{C}_{6} \int_{\tau_{0}}^{\tau} \mathrm{e}^{-2 \gamma(\tau-t)}\|s(t)\|_{\mathrm{H}^{2}}^{2} \mathrm{~d} t \leq \bar{C}_{7} \mathrm{e}^{-2 \gamma \tau}
$$

hence $\|\psi(\tau)\|_{\mathrm{H}^{2}}=\mathcal{O}\left(\mathrm{e}^{-\gamma \tau}\right)$ as $\tau \rightarrow+\infty$. The proof of Theorem 4.1 is now complete.

\section{References}

[BrK92] J. Bricmont, A. Kupiainen. Renormalization group and the GinzburgLandau equation. Commun. Math. Physics 150 (1992) 193-208.

[CL55] E. Coddington, N. Levinson. Theory of ordinary differential equations. McGraw-Hill, New-York, 1955.

[Col94] P. Collet. Thermodynamic limit of the Ginzburg-Landau equations. Nonlinearity 7 (1994) 1175-1190.

[CoE90a] P. Collet, J.-P. Eckmann. Instabilities and fronts in extended systems. Princeton University Press, 1990.

[CoE90b] P. Collet, J.-P. Eckmann. The time-dependent amplitude equation for the Swift-Hohenberg problem. Commun. Math. Phys. 132 (1990) 139-153.

[CoE92] P. Collet, J.-P. Eckmann. Solutions without phase-slip for the GinsburgLandau equation. Commun. Math. Physics 145 (1992) 345-356.

[CEE92] P. Collet, J.-P. Eckmann, H. Epstein. Diffusive repair for the GinsburgLandau equation. Helv. Phys. Acta 65 (1992) 56-92.

[Eck65] W. Eckhaus. Studies in non-linear stability theory. Springer tract in Nat. Phil. 6, Springer, 1965.

[EWW98] J.-P. Eckmann, C.E. Wayne, P. Wittwer. Geometric stability analysis of periodic solutions of the Swift-Hohenberg equation. Commun. Math. Physics, to appear 1998.

[Ga95] Th. Gallay. Periodic patterns and traveling fronts for the Ginzburg-Landau equation. Proceedings of the IUTAM/ISIMM Symposium on Structure and $d y$ namics of nonlinear waves in fluids held in Hannover 1994, World Scientific, 1995.

[GR97] Th. Gallay, G. Raugel. Scaling variables and asymptotic expansions in damped wave equations. Prépublication de l'Université de Paris-Sud no. 97-66, 1997.

[He81] D. Henry. Geometric theory of semilinear parabolic equations. Lecture Notes in Mathematics 840, Springer, Berlin, 1981. 
[KSM92] P. Kirrmann, G. Schneider, A. Mielke. The validity of modulation equations for extended systems with cubic nonlinearities. Proc. Royal Soc. Edinburgh 122A (1992) 85-91.

[Mi97a] A. Mielke. The complex Ginzburg-Landau equation on large and unbounded domains: sharper bounds and attractors. Nonlinearity 10 (1997) 199-222.

[Mi97b] A. Mielke. Mathematical analysis of sideband instabilities with application to Rayleigh-Bénard convection. J. Nonlinear Science 7 (1997) 57-99.

[Mi97c] A. Mielke. Instability and stability of rolls in the Swift-Hohenberg equation. Commun. math. Physics 189 (1997) 829-853.

[MiS95] A. Mielke, G. Schneider. Attractors for modulation equations on unbounded domains - existence and comparison. Nonlinearity 8 (1995) 1-26.

[RS72] M. Reed, B. Simon. Methods of modern mathematical physics vol. IV : Analysis of operators. Academic Press, New-York, 1972.

[Sch96] G. Schneider. Diffusive stability of spatial periodic solutions of the SwiftHohenberg equation. Commun. Math. Physics 178 (1996) 679-702.

[Sch97] G. Schneider. Nonlinear stability of Taylor vortices in infinite cylinders. Habilitation Thesis, Universität Hannover, 1997. Submitted to Arch. Rational Mech. Analysis.

[Uec98] H. Uecker. Diffusive stability of roll solutions in the two-dimensional SwiftHohenberg equation. Preprint Universität Hannover, Institut für Angewandte Mathematik, January 1998.

[Way97] C.E. Wayne. Invariant manifolds for parabolic partial differential equations on unbounded domains. Archive Rat. Mech. Analysis 138 (1997) 279-306.

[Zei90] E. Zeidler. Nonlinear Functional Analysis and Its Applications II/B. SpringerVerlag, 1990. 